\title{
QUASISYMMETRIC FUNCTIONS FROM A TOPOLOGICAL POINT OF VIEW
}

\author{
ANDREW BAKER and BIRGIT RICHTER*
}

\begin{abstract}
It is well-known that the homology of the classifying space of the unitary group is isomorphic to the ring of symmetric functions Symm. We offer the cohomology of the space $\Omega \Sigma C P^{\infty}$ as a topological model for the ring of quasisymmetric functions QSymm. We exploit standard results from topology to shed light on some of the algebraic properties of QSymm. In particular, we reprove the Ditters conjecture. We investigate a product on $\Omega \Sigma C P^{\infty}$ that gives rise to an algebraic structure which generalizes the Witt vector structure in the cohomology of $B U$. The canonical Thom spectrum over $\Omega \Sigma C P^{\infty}$ is highly non-commutative and we study some of its features, including the homology of its topological Hochschild homology spectrum.
\end{abstract}

\section{Introduction}

Let us recall some background on the variants of symmetric functions. For a much more detailed account on that see [17], [18].

The algebra of symmetric functions, Symm, is an integral graded polynomial algebra

$$
\text { Symm }=\mathrm{Z}\left[c_{1}, c_{2}, \ldots\right],
$$

where $c_{i}$ has degree $2 i$. The reader is encouraged to think of these $c_{i}$ as Chern classes. This algebra structure can be extended to a Hopf algebra structure by defining the coproduct to be that given by the Cartan formula

$$
\Delta\left(c_{n}\right)=\sum_{p+q=n} c_{p} \otimes c_{q} .
$$

The antipode on Symm is defined as

$$
\chi\left(c_{n}\right)=\sum_{i_{1}+\cdots+i_{m}=n}(-1)^{m} c_{i_{1}} \cdot \ldots \cdot c_{i_{m}} .
$$

* The authors thank the Mittag-Leffler institute for support and hospitality allowing major parts of this work to be carried out during their stay there. The first-named author was supported by the Carnegie Trust for the Universities of Scotland.

Received April 17, 2007; in revised form August 13, 2007. 
This Hopf algebra is self dual in the sense that there is an isomorphism of Hopf algebras

$$
\text { Symm* } \cong \text { Symm, }
$$

where Symm* is the degree-wise Z-linear dual of Symm. In particular, Symm is bipolynomial, i.e., the underlying algebra of the Hopf algebra and its dual are both polynomial algebras.

The non-commutative analogue of the algebra Symm is the algebra of nonsymmetric functions, NSymm (also known as the Leibniz algebra) which is the free associative graded $Z$-algebra $Z\left\langle Z_{1}, Z_{2}, \ldots\right\rangle$ on generators $Z_{1}, Z_{2}, \ldots$, where $Z_{i}$ has degree $2 i$. Again, NSymm comes with a natural coproduct given by

$$
\Delta\left(Z_{n}\right)=\sum_{p+q=n} Z_{p} \otimes Z_{q}
$$

and an antipode given on $Z_{n}$ by

$$
\chi\left(Z_{n}\right)=\sum_{a_{1}+\cdots+a_{m}=n}(-1)^{m} Z_{a_{1}} \cdot \ldots \cdot Z_{a_{m}} .
$$

The Hopf algebra of quasisymmetric functions (sometimes written quasisymmetric functions), QSymm, is defined to be the dual Hopf algebra to NSymm. We follow the convention from [17], [18], denoting the element dual to the monomial $Z_{a_{1}} \cdot \ldots \cdot Z_{a_{n}}$ with respect to the monomial basis by $\alpha=\left[a_{1}, \ldots, a_{n}\right]$ and call the number $a_{1}+\cdots+a_{n}$ the degree of $\alpha$. The resulting product structure among these elements is given by the overlapping shuffle product of [16, section 3]. For example, using the dual pairing, we find that

$$
[3][1,2]=[3,1,2]+[1,3,2]+[1,2,3]+[4,2]+[1,5] .
$$

Often it is useful to vary the ground ring and replace the integers by some other commutative ring with unit $R$. We define $\operatorname{Symm}(R)$ to be $\operatorname{Symm} \otimes R$, and similarly we set $\operatorname{NSymm}(R)=\operatorname{NSymm} \otimes R$ and QSymm $(R)=\mathrm{QSymm} \otimes R$.

The algebras NSymm and QSymm have received a great deal of attention in combinatorics. Several structural properties were proven, for instance about the explicit form of the primitives in the coalgebra NSymm [19] or the freeness of QSymm as a commutative algebra [16]. The latter result is known as the Ditters conjecture, and is our Theorem 2.1. The original methods of proof came from within combinatorics. We offer an alternative proof using ingredients from algebraic topology.

In the case of symmetric functions, Liulevicius [21] exploited the identification of Symm with the cohomology of $B U$ to use topology to aid the understanding of some of the properties of Symm. In this paper we offer a 
topological model for the Hopf algebras NSymm and QSymm by interpreting them as homology and cohomology of the loop space of the suspension of the infinite complex projective space, $\Omega \Sigma C P^{\infty}$.

Our desire to find a topological model for the algebra of quasisymmetric functions has its origin in trying to understand Jack Morava's thoughts on connecting Galois theory of structured ring spectra to motivic Galois theory, as explained in [25]. We do not claim that our insights are helpful in this context, but it was our motivation to start this investigation.

The first part of this paper is concerned with the algebraic structure of the algebra of quasisymmetric functions.

In Section 1 we describe the isomorphism between the Hopf algebra NSymm and the homology of the loop space on the suspension of the infinite complex projective space. This identification is probably known to many people, but we do not know of any source where this is seriously exploited.

We give a proof of the Ditters conjecture in Section 2. This conjecture states that the algebra QSymm is polynomial and was proven by Hazewinkel in [16]. Our proof uses the Hilton-Milnor theorem which also yields an explicit set of generators over the rationals.

The second part deals with the topological properties of the model $\Omega \Sigma C P^{\infty}$ and its relation to $B U$.

We investigate the $p$-local structure of QSymm in Section 3 using the splitting of $\Sigma \mathrm{C} P^{\infty}$ at a prime $p$. We discuss Steenrod operations on QSymm $\left(\mathrm{F}_{p}\right)$ in Section 4.

It is well-known that the ring of big Witt vectors on a commutative ring is represented by Symm. Topologically this structure is induced by the two canonical $H$-structures on $B U$. We recall this in Section 5, then in Section 6 we introduce product structures on $\Omega \Sigma C P^{\infty}$ which in cohomology produces a structure that we call the quasi-Witt structure on QSymm, a hitherto unremarked algebraic structure, which differs from the one explored by Hazewinkel in [17, $\S 14]$.

The canonical map from $\Omega \Sigma C P^{\infty}$ to $B U$ is a loop map. Therefore the associated Thom spectrum has a strictly associative multiplication. But as is visible from the non-commutativity of its homology, it is not even homotopy commutative. We will describe some of its features in Sections 7 and investigate the homology of its topological Hochschild homology spectrum in Section 8. 


\section{Part 1. Algebraic properties of the algebra of quasisymmetric functions}

\section{A topological manifestation of NSymm}

In this part we will recall some standards facts about $H_{*}\left(\Omega \Sigma C P^{\infty}\right)$.

There is a nice combinatorial model $J X$ for any topological space of the form $\Omega \Sigma X$ with $X$ connected, namely the James construction on $X$, fully described in [34, VII §2]. After one suspension this gives rise to a splitting

$$
\Sigma \Omega \Sigma X \sim \Sigma J X \sim \bigvee_{n \geqslant 1} \Sigma X^{(n)},
$$

where $X^{(n)}$ denotes the $n$-fold smash power of $X$.

If the homology of $X$ is torsion-free, then the homology of $J X$ is the tensor algebra on the reduced homology of $X$

$$
H_{*}(J X) \cong T\left(\widetilde{H}_{*}(X)\right) .
$$

The concatenation of loops in $\Omega \Sigma C P^{\infty}$ together with the diagonal on $\Omega \Sigma C P^{\infty}$ turns the homology of $\Omega \Sigma C P^{\infty}$ into a Hopf algebra.

The integral homology of $\mathrm{C} P^{\infty}$ has $H_{i}\left(\mathrm{C} P^{\infty}\right)=\mathrm{Z}$ for all even $i$ with generators $\beta_{i} \in H_{2 i}\left(\mathrm{C} P^{\infty}\right)$ and is trivial in odd degrees. Therefore

$$
H_{*}\left(\Omega \Sigma \mathrm{C} P^{\infty}\right) \cong T\left(\widetilde{H}_{*}\left(\mathrm{C} P^{\infty}\right)\right)=\mathrm{Z}\left\langle\beta_{1}, \beta_{2}, \ldots\right\rangle,
$$

with $\beta_{i}$ being a non-commuting variable in degree $2 i$. Thus there is an isomorphism of algebras

$$
H_{*}\left(\Omega \Sigma \mathrm{C} P^{\infty}\right) \cong \mathrm{NSymm}
$$

under which $\beta_{n}$ corresponds to $Z_{n}$. The coproduct $\Delta$ on $H_{*}\left(\Omega \Sigma C P^{\infty}\right)$ induced by the diagonal in $\Omega \Sigma C P^{\infty}$ is compatible with the one on NSymm:

$$
\Delta\left(\beta_{n}\right)=\sum_{p+q=n} \beta_{p} \otimes \beta_{q} .
$$

Putting this information together, we see that (1.2) gives an isomorphism of graded, connected Hopf algebras. Note that the antipode $\chi$ in $H_{*}\left(\Omega \Sigma C P^{\infty}\right)$ arises from the time-inversion of loops. As antipodes are unique for Hopf algebras which are commutative or cocommutative, this gives a geometric interpretation for the antipode in NSymm.

As the homology of $\Omega \Sigma C P^{\infty}$ is a graded free abelian group, the linear dual of $H_{*}\left(\Omega \Sigma C P^{\infty}\right)$ is canonically isomorphic to the cohomology, $H^{*}\left(\Omega \Sigma C P^{\infty}\right)$, which is also a Hopf algebra. Thus we have proven the following result. 
THEOREM 1.1. There are isomorphisms of graded Hopf algebras

$$
H_{*}\left(\Omega \Sigma \mathrm{C} P^{\infty}\right) \cong \mathrm{NSymm}, \quad H^{*}\left(\Omega \Sigma \mathrm{C} P^{\infty}\right) \cong \mathrm{QSymm} .
$$

REMARK 1.2. Note that the cohomological degree of a generator corresponding to a sequence $\alpha=\left[a_{1}, \ldots, a_{n}\right]$ is twice its degree.

There is the canonical inclusion map $j: C P^{\infty}=B U(1) \longrightarrow B U$. The universal property of $\Omega \Sigma \mathrm{C} P^{\infty}$ as a free $H$-space gives an extension to a loop map

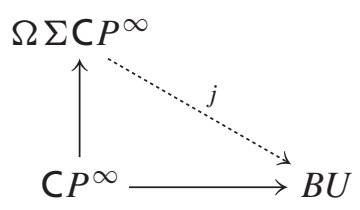

which induces a virtual bundle $\xi$ on $\Omega \Sigma C P^{\infty}$. On homology, the map $j$ induces an epimorphism because $C P^{\infty}$ gives rise to the algebra generators in $H_{*}(B U)$ and correspondingly, $H^{*}(j)$ is a monomorphism on cohomology. This corresponds to the inclusion of symmetric functions into quasisymmetric functions. We will describe this in more detail in Section 5.

Hazewinkel mentions in [19] that over the rationals the Lie algebra of primitives in the Hopf algebra NSymm(Q) is free and says that the primitive part of NSymm "is most definitely not a free Lie algebra; rather it tries to be something like a divided power Lie algebra (though I do not know what such a thing would be)". In this section we give a topological proof of the rational result and we explain how to make sense of this last comment in positive characteristic.

A theorem of Milnor and Moore [24, Appendix] identifies the Lie-algebra of primitives in the Hopf algebra $H_{*}\left(\Omega \Sigma C P^{\infty}\right.$; Q $)$ with the Lie-algebra $\pi_{*}\left(\Omega \Sigma C P^{\infty}\right) \otimes \mathrm{Q}$. Here the Lie-algebra structure on $\pi_{*}\left(\Omega \Sigma C P^{\infty}\right)$ is that given by the Samelson-Whitehead product [34, X §§5-7].

Let $C$ be a simply connected rational co- $H$-space that is a CW-complex. Scheerer in [29, pp. 72-73] proves that for such spaces $C$, the Lie algebra $\pi_{*}(\Omega C)$ is a free Lie-algebra. So in particular, $\pi_{*}\left(\Omega \Sigma C P^{\infty}\right) \otimes \mathrm{Q}$ is a free Lie-algebra.

In the case of positive characteristic, operads help to identify the primitives in NSymm. In [12, theorem 1.2.5], Fresse uses the fact that for a vector space $V$ over a field $k$ of characteristic $p$, the primitives in a tensor algebra $T(V)$ can be identified with the free $p$-restricted Lie-algebra generated by $V$. He shows that the free $p$-restricted Lie-algebra is isomorphic to the direct sum of invariants

$$
\bigoplus_{n \geqslant 1}\left(\operatorname{Lie}(n) \otimes V^{\otimes n}\right)^{\Sigma_{n}}
$$


where $\operatorname{Lie}(n)$ is the $n$th part of the operad which codifies Lie algebras. We note that instead of invariants we may take coinvariants

$$
\bigoplus_{n \geqslant 1}\left(\operatorname{Lie}(n) \otimes V^{\otimes n}\right)_{\Sigma_{n}}
$$

to give the free Lie algebra generated by the vector space $V$, whereas in Fresse's terminology of [12, p. 4122], the invariants codify the free Lie algebra with divided symmetries. In the case when $V$ is the vector space generated by $Z_{1}, Z_{2}, \ldots$ we may deduce the following result.

Proposition 1.3. For a field $k$ of positive characteristic $p$, the Lie subalgebra of primitives $\operatorname{Prim}(\mathrm{NSymm}(k))$ agrees with the free p-restricted Liealgebra on the $k$-vector space $V$ generated by $Z_{1}, Z_{2}, \ldots$ and furthermore there is an isomorphism

$$
\bigoplus_{n \geqslant 1}\left(\operatorname{Lie}(n) \otimes V^{\otimes n}\right)^{\Sigma_{n}} \cong \operatorname{Prim}(\operatorname{NSymm}(k)) .
$$

\section{A proof of the Ditters conjecture}

In this section we give a topological proof of the Ditters conjecture which asserts that the algebra QSymm is a free commutative algebra (see [16], [18]), and use the Hilton-Milnor theorem to show that over the rationals the generators can be indexed on Lyndon words (see Definition 2.5). The Ditters conjecture started off as a statement [9, proposition 2.2], but it turned out that the proof was not correct. There were later attempts by Ditters and Scholtens to prove the conjecture [10], however, the line of argument there turned out to be incorrect as well. Hazewinkel proved the conjecture in [16]. For another approach on related matters from a topological perspective see [8]. Here is our statement of these results.

THEOREM 2.1. The algebra of quasisymmetric functions, QSymm, is a free commutative algebra. Over the rationals, the polynomial generators of $\mathrm{QSymm}(\mathrm{Q})$ in degree $2 n$ can be indexed on Lyndon words of degree $n$.

This Theorem recovers Hazewinkel's result [16, theorem 8.1]. Recall that the degree of a word $a_{1} \cdot \ldots \cdot a_{n}$ with $a_{i} \in \mathrm{N}$ is $a_{1}+\cdots+a_{n}$.

Our proof proceeds by using Borel's theorem on the structure of Hopf algebras over perfect fields [24, theorem 7.11] to first identify the rationalization of QSymm as a polynomial algebra and then to show that the $\mathrm{F}_{p}$-reductions are polynomial for all primes $p$. Finally, we use a gluing result Proposition 2.4 to obtain the integral statement. For the explicit form of the generators we 
compare the words arising in the Hilton-Milnor theorem to Lyndon words in Proposition 2.6.

The rational version of Borel's theorem immediately implies that the algebra of rational quasisymmetric functions, QSymm(Q), is a polynomial algebra because all its generators live in even degrees.

Rationally the suspension of $C P^{\infty}$ splits into a wedge of rational spheres

$$
\Sigma \mathrm{C} P_{\mathrm{Q}}^{\infty} \sim \mathrm{S}_{\mathrm{Q}}^{3} \vee \mathrm{S}_{\mathrm{Q}}^{5} \vee \cdots \sim \Sigma\left(\mathrm{S}_{\mathrm{Q}}^{2} \vee \mathrm{S}_{\mathrm{Q}}^{4} \vee \cdots\right) .
$$

Therefore we can apply the Hilton-Milnor theorem for loops on the suspension of a wedge of spaces [33, theorem 1.2] and obtain

$$
\Omega \Sigma\left(\mathrm{S}_{\mathrm{Q}}^{2} \vee \mathrm{S}_{\mathrm{Q}}^{4} \vee \cdots\right) \sim \prod_{\alpha}^{\prime} \Omega N_{\alpha},
$$

where after suitable suspension, $N_{\alpha}$ is a smash product of rational spheres, thus its cohomology is monogenic polynomial. The $\alpha$ in the indexing set of the weak product run over all basic products in the sense of Whitehead [34, pp. 511-512].

Note that in the usual formulation of the Hilton-Milnor theorem, only a finite number of wedge summands are considered. However, a colimit argument gives the countable case as well. In Subsection 2.2 we give a bijection between the set of basic products and the set of Lyndon words. Thus

$$
\mathrm{QSymm}(\mathrm{Q}) \cong \mathrm{Q}\left[x_{\alpha} \mid \alpha \text { Lyndon }\right] \text {. }
$$

Now we consider the $\mathrm{F}_{p}$-cohomology $H^{*}\left(\Omega \Sigma \mathrm{C} P^{\infty} ; \mathrm{F}_{p}\right)$ which is canonically isomorphic to the mod $p$ reduction of the quasisymmetric functions, QSymm $\left(\mathrm{F}_{p}\right)$. A priori the Borel theorem allows for truncated polynomial algebras. However we will use the action of the Steenrod algebra to prove:

PROPOSITION 2.2. QSymm $\left(\mathrm{F}_{p}\right)$ is a polynomial algebra.

Proof. Using the James splitting we obtain that as a module over the Steenrod algebra $\mathscr{A}_{p}^{*}$, the positive part of QSymm $\left(\mathrm{F}_{p}\right)$ has a direct sum decomposition

$$
\operatorname{QSymm}\left(\mathrm{F}_{p}\right)^{*>0} \cong \bigoplus_{n} \widetilde{H}^{*}\left(\left(\mathrm{C} P^{\infty}\right)^{(n)} ; \mathrm{F}_{p}\right) .
$$

We have to show that no $p$ th power of an element $x$ can be zero. Such a power corresponds to the Steenrod operation $\mathscr{P P}|x| / 2$ applied to $x$. If $p$ is odd, we write $\mathscr{P}^{i}$ for the reduced power operation, while for $p=2$, we set $\mathscr{P}^{i}=S q^{2 i}$.

These operations are non-trivial on the cohomology of $C P^{\infty}$ and from the Cartan formula we see that they are non-trivial on the cohomology of the smash powers. 
Remark 2.3. The above proof showed that the $p$ th power operation on the algebra QSymm $\left(\mathrm{F}_{p}\right)$ is non-trivial. We will explicitly determine the action of the mod $p$ Steenrod algebra on QSymm $\left(\mathrm{F}_{p}\right)$ in Section 4.

Taking the rational result and the $\mathrm{F}_{p}$-cohomology result together with Proposition 2.4 below yields a proof of Theorem 2.1.

\subsection{An auxiliary result on polynomial algebras}

In this section we provide a useful local to global result on polynomial algebras which may be known but we were unable to locate a specific reference.

If $R$ is a commutative ring, then for a graded $R$-algebra $A^{*}$, we write $D A^{n}$ (resp. $Q A^{n}$ ) for the decomposables (resp. indecomposables) in degree $n$. Unspecified tensor products will be taken over whatever ground ring $R$ is in evidence. If $p$ is a positive prime or 0 , let $\mathbf{F}_{p}$ denote either the corresponding Galois field or $\mathrm{F}_{0}=\mathrm{Q}$.

Proposition 2.4. Let $H^{*}$ be a graded commutative connective Z-algebra which is concentrated in even degrees and with each $H^{2 n}$ a finitely generated free abelian group. If for each non-negative rational prime $p, H(p)^{*}=H^{*} \otimes$ $\mathrm{F}_{p}$ is a polynomial algebra, then $H^{*}$ is a polynomial algebra and for every non-negative rational prime $p$,

$$
\text { rank } Q H^{2 n}=\operatorname{dim}_{\mathrm{F}_{p}} Q H(p)^{2 n} .
$$

Proof. Let $p \geqslant 0$ be a prime. We will denote by $\pi_{p}(n)$ the number of polynomial generators of $H(p)^{*}$ in degree $2 n$, and this is equal to $\operatorname{dim}_{\mathrm{F}_{p}} Q H(p)^{2 n}$. The Poincaré series of the even degree part of $H^{*}(p)$ satisfies

$$
\sum_{n \geqslant 0} \operatorname{rank} H^{2 n} t^{n}=\sum_{n \geqslant 0} \operatorname{rank} H(p)^{2 n} t^{n}=\prod_{n \geqslant 0}\left(1-t^{n}\right)^{-\pi_{p}(n)},
$$

hence $\pi_{p}(n)$ is independent of $p$.

Now it is easy to see that the natural homomorphism $D H^{2 n} \otimes \mathrm{Q} \longrightarrow$ $D H(0)^{2 n}$ is an isomorphism and therefore the natural homomorphism $Q H^{2 n} \otimes$ $\mathrm{Q} \longrightarrow Q H(0)^{2 n}$ is an isomorphism. Furthermore, for each positive prime $p$ there is a commutative diagram

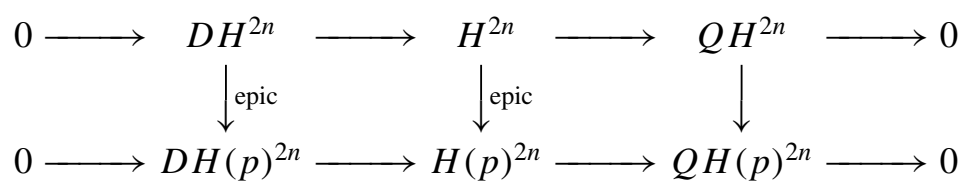


with exact rows and whose columns have the indicated properties. Since the right hand vertical homomorphism factors as

$$
Q H^{2 n} \longrightarrow Q H^{2 n} \otimes \mathrm{F}_{p} \longrightarrow Q H(p)^{2 n}
$$

we see that the right hand factor is an epimorphism $Q H^{2 n} \otimes \mathrm{F}_{p} \longrightarrow Q H(p)^{2 n}$. This implies that

$$
\pi_{0}(n) \geqslant \pi_{p}(n) .
$$

We will now show that the indecomposables in degree $2 n$ are torsion free. Assume that $Q H^{2 n}$ were of the form

$$
Q H^{2 n}=\mathrm{Z}^{r} \oplus \bigoplus_{p} T_{p}
$$

where $p$ runs over a finite set of primes and $T_{p}$ is the $p$-torsion subgroup of $Q H^{2 n}$. Then

$$
Q H^{2 n} \otimes \mathrm{F}_{p} \cong \mathrm{F}_{p}^{r} \oplus T_{p} \otimes \mathrm{F}_{p} .
$$

Thus if $T_{p} \neq 0$, then the dimension of $Q H^{2 n} \otimes \mathrm{F}_{p}$ as an $\mathrm{F}_{p}$-vector space would be strictly bigger than $r$. However, the following argument shows that these dimensions are equal and so $T_{p}$ has to be zero.

Let $I=H^{*>0}$ and $I_{\mathrm{F}_{p}}=H^{*>0} \otimes \mathrm{F}_{p}=I \otimes \mathrm{F}_{p}$. Observe that

$$
I \otimes \mathrm{F}_{p} \otimes I \otimes \mathrm{F}_{p} \cong(I \otimes I) \otimes \mathrm{F}_{p}
$$

and consider the following commuting diagram with exact rows.

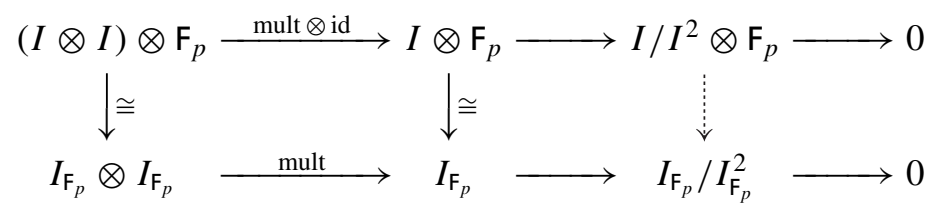

Thus we obtain that $I / I^{2} \otimes \mathrm{F}_{p} \cong I_{\mathrm{F}_{p}} / I_{\mathrm{F}_{p}}^{2}$, i.e., $Q H^{2 n} \otimes \mathrm{F}_{p} \cong Q H(p)^{2 n}$.

Now for each $n$, choose a lifting of a basis of $Q H(p)^{2 n}$ to linearly independent elements $x_{n, i}$ of $H^{2 n}$. It is clear that under the natural monomorphism $H^{2 n} \longrightarrow H(0)^{2 n}$, these give a part of a polynomial generating set for $H(0)^{2 n}$ and therefore generate a polynomial subalgebra $P^{*}=\mathrm{Z}\left[x_{n, i}: n, i\right] \subseteq H^{*}$. We will use induction on degree to show that we have equality here.

For $n=1$, we have $Q H^{2}=H^{2}$. Now suppose that we have $H^{*}=P^{*}$ in degrees less than $2 k$. Then $D H^{2 k}=D P^{2 k}$ and for each positive prime $p$ there 
is a diagram with short exact rows

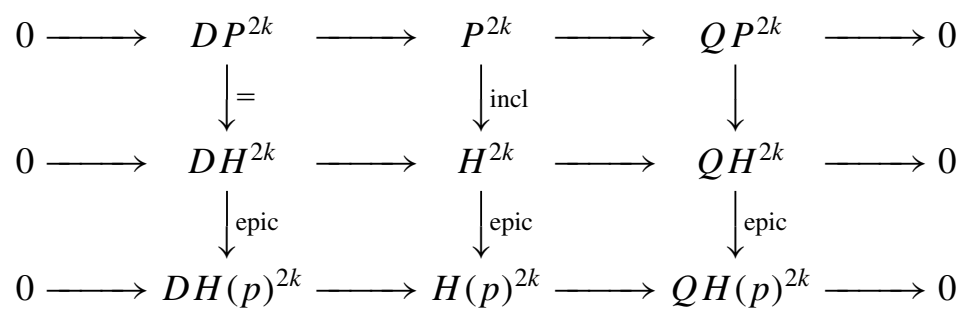

in which the composite homomorphism $P^{2 k} \longrightarrow Q H(p)^{2 k}$ is surjective. To complete the proof, we must show that the cokernel of the inclusion $P^{2 k} \longrightarrow$ $H^{2 k}$ is trivial. Since $P^{2 k}$ and $H^{2 k}$ agree rationally, this cokernel is torsion and it suffices to verify this locally at each prime $p$. The map from $P^{2 k} \otimes \mathrm{F}_{p}$ to $H(p)^{2 k}$ is an isomorphism, so we see that over the local ring $\mathbf{Z}_{(p)}$,

$$
P_{(p)}^{2 k}+p H_{(p)}^{2 k}=H_{(p)}^{2 k}
$$

and Nakayama's Lemma implies that $P_{(p)}^{2 k}=H_{(p)}^{2 k}$. Thus $P^{2 k}=H^{2 k}$ and we have established the induction step.

\subsection{Basic products and Lyndon words}

Usually [9], [16] the set of polynomial generators of the rationalized algebra of quasisymmetric functions is indexed on Lyndon words, whereas our approach yields a polynomial basis indexed on basic products in the sense of [34, pp. 511512]. The aim of this section is to compare these two sets of generators.

First, let us recall some notation and definitions. See [28, §5] for more details.

Let $A$ be an alphabet, finite or infinite. We assume that $A$ is linearly ordered. The elements of $A$ are called letters. Finite sequences $a_{1} \ldots a_{n}$ with $a_{i} \in A$ are words; the number of letters in a word is its length. We use the lexicographical ordering on words given as follows. A word $u$ is smaller than a word $v$ if and only if $v=u r$, where $r$ is a non-empty word or if $u=w a u^{\prime}$ and $v=w b v^{\prime}$ where $w, u^{\prime}, v^{\prime}$ are words and $a$ and $b$ are letters with $a<b$. If a word $w$ can be decomposed as $w=u v$, then $v$ is called a right and $u$ is called the left factor. If $u$ is not the empty word, then $v$ is called a proper right factor.

Definition 2.5. A word is Lyndon if it is a non-empty word which is smaller than any of its proper right factors.

So for example, if $A$ is the alphabet that consists of the natural numbers with its standard ordering, then the first few Lyndon words are

$$
1,2,3, \ldots, 12,13,23, \ldots, 112, \ldots
$$


Words are elements in the free monoid generated by the alphabet $A$. Let $M(A)$ be the free magma generated by $A$, i.e., we consider non-associative words built from the letters in $A$. Elements in the free magma correspond to planar binary trees with a root where the leaves are labelled by the elements in $A$. For instance, if $a, b, c$ are letters, then the element $(a b) c$ corresponds to the tree

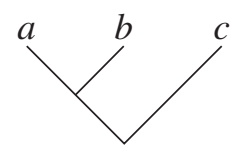

whereas $a(b c)$ corresponds to

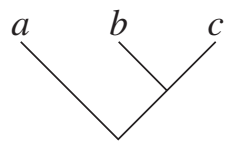

We will briefly recall the construction of basic products. For more details see [34].

Let $A$ be an alphabet whose letters are linearly ordered, for instance $A=$ $\left\{a_{1}, \ldots, a_{k}\right\}$ with $a_{1}<\ldots<a_{k}$. Basic products of length one are just the letters $a_{i}$. We assign a rank and a serial number to each basic product. The convention for basic products of length one is that the serial number of $a_{i}$ is $s\left(a_{i}\right)=i$, whereas the rank is $r\left(a_{i}\right)=0$.

Assume that basic products of length up to $n-1$ have been already defined and that these words are linearly ordered in such a way that a word $w_{1}$ is less than a word $w_{2}$ if the length of $w_{1}$ is less than the length of $w_{2}$, and assume that we have assigned ranks to all those words. Then the basic products of length $n$ are all (non-associative) words of length $n$ of the form $w_{1} w_{2}$ such that the $w_{i}$ are basic products, $w_{2}<w_{1}$ and the rank of $w_{1}, r\left(w_{1}\right)$, is smaller than the serial number of $w_{2}, s\left(w_{2}\right)$. We choose an arbitrary linear ordering on these products of length $n$ and we define their rank as $r\left(w_{1} w_{2}\right)=s\left(w_{2}\right)$.

Any basic product on $A$ is in particular an element of the free magma generated by $A$. We need the fact that on an alphabet with $k$ elements, the number of basic products of length $n$ is

$$
\frac{1}{n} \sum_{d \mid n} \mu(d) k^{n / d},
$$

where $\mu$ is the Möbius function [34, p. 514]. This number agrees with the number of Lyndon words of length $n$ on such an alphabet, see [28, theorem 5.1, 
\& corollary 4.14] for details. So we know that there is an abstract bijection between the two sets.

Proposition 2.6. There is a canonical algorithm defining a bijection between the set of basic products and the set of Lyndon words on a finite alphabet $A$.

Roughly speaking, the idea of the proof is to 'correct' the ordering in the product $w_{1} w_{2}$ of basic products with $w_{2}<w_{1}$ and to switch the product back to one that is ordered according to the convention used for building Lyndon words.

Proof. We start with a basic product $\xi$ of length $n$ and consider it as an element in the magma generated by $A$ and take its associated planar binary tree. A binary tree has a natural level structure: we regard a binary tree such as

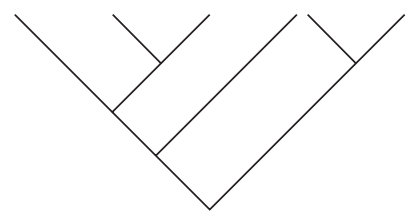

as having four levels



Starting with a basic product of length $n$, its tree has some number of levels, say $m$. The idea is to work from the leaves of the tree to its root and transform the basic product into a Lyndon word during this process.

We start with level $m$. Whenever there is a part of a tree of $\mathrm{V}$-shape we induce a multiplication; otherwise we leave the element as it is. In the above example, the starting point could be a word such as $\left(\left(a_{1}\left(a_{2} a_{3}\right)\right) a_{4}\right)\left(a_{5} a_{6}\right)$.

From level $m$ to level $m-1$ we induce multiplication whenever there is a local picture like

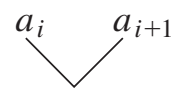


and in such a case we send $\left(a_{i} a_{i+1}\right)$ to

$$
\begin{cases}a_{i} a_{i+1} & \text { if } a_{i}<a_{i+1}, \\ a_{i+1} a_{i} & \text { if } a_{i}>a_{i+1} .\end{cases}
$$

Note that in a basic product equal neighbours $a_{i}=a_{i+1}$ do not occur. We place this product on the corresponding leaf in level $m-1$.

Iterating this procedure gives a reordered word in the alphabet $A$.

We have to prove that this is a Lyndon word. We do this by showing that in each step in the algorithm we produce Lyndon words. Starting with the highest level $m$ this is clear because words of length two of the form $a b$ with $a<b$ are Lyndon words.

In the following we will slightly abuse notation and use $\prod_{j=1}^{t} a_{i}$ for the ordered product $a_{1} \cdot \ldots \cdot a_{t}$. Assume that we have reduced the tree down to an intermediate level less than $m$ and obtained Lyndon words as labels on the leaves. In the next step we have to check that every multiplication on subtrees of the form

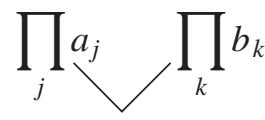

with $\prod_{j=1}^{t} a_{j}$ and $\prod_{k=1}^{s} b_{k}$ Lyndon words, again give a Lyndon word. But this is proved in [28, (5.1.2), p. 106].

Each step in the algorithm is reversable, therefore the algorithm defines an injective map. As the cardinalities of the domain and target agree, this map is a bijection.

\section{Part 2. $\Omega \Sigma C P^{\infty}$ : a splitting, Witt vectors and its associated Thom spectrum}

\section{A $p$-local splitting}

In this section we fix an odd prime $p$. On the one hand, from [1, lecture 4] we have the Adams splitting of $B U$ localized at $p$,

$$
B U_{(p)} \simeq W_{1} \times \cdots \times W_{p-1}
$$

which is a splitting of infinite loop spaces. On the other hand, by [23] there is an unstable $p$-local splitting of $\Sigma C P_{(p)}^{\infty}$ into a wedge of spaces,

$$
\Sigma \mathrm{C} P_{(p)}^{\infty} \simeq Y_{1} \vee \cdots \vee Y_{p-1}
$$


where the bottom cell of $Y_{i}$ is in degree $2 i+1$.

REMARK 3.1. Each space $Y_{i}$ inherits a co- $H$-space structure from $\Sigma \mathrm{C} P_{(p)}^{\infty}$ via the inclusion and projection maps. However, this co- $H$-structure is not necessarily co-associative and neither are the inclusion and projection maps necessarily co- $H$-maps. Furthermore, each $Y_{i}$ is minimal atomic in the sense of [5], and cannot be equivalent to a suspension except in the case $i=p-1$ when it does desuspend [14].

Recent work of Selick, Theriault and Wu [31] establishes a Hilton-Milnor like splitting of loops on such a wedge of co- $H$-spaces. In our case, the splitting is of the familiar form

$$
\Omega \Sigma C P_{(p)}^{\infty} \simeq \Omega\left(Y_{1} \vee \cdots \vee Y_{p-1}\right) \simeq \prod_{\alpha}^{\prime} \Omega N_{\alpha},
$$

where $\alpha$ runs over all basic products formed on the alphabet $\{1, \ldots, p-1\}$ and $\prod^{\prime}$ denotes the weak product. In cohomology, i.e., in $\operatorname{QSymm}\left(Z_{(p)}\right)$, this splitting gives rise to a splitting of algebras,

$$
H^{*}\left(\Omega \Sigma C P_{(p)}^{\infty}\right) \cong \bigotimes_{\alpha} H^{*}\left(\Omega N_{\alpha}\right)
$$

If $\nu_{i}(\alpha)$ denotes the number of occurrences of the letter $i$ in the word $\alpha$, then

$$
\Sigma^{v_{1}(\alpha)+\cdots+v_{p-1}(\alpha)-1} N_{\alpha} \simeq Y_{1}^{\left(v_{1}(\alpha)\right)} \wedge \cdots \wedge Y_{p-1}^{\left(v_{p-1}(\alpha)\right)}
$$

where $X^{(n)}$ denotes the $n$th smash power of $X$. The homology of the space $Y_{i}$ starts with a generator in degree $2 i+1$, thus the smash power $Y_{1}^{\left(v_{1}(\alpha)\right)} \wedge$ $\cdots \wedge Y_{p-1}^{\left(v_{p-1}(\alpha)\right)}$ has homology starting in degree $\sum(2 i+1) \nu_{i}(\alpha)$. Thus $\Omega N_{\alpha}$ has bottom degree $2 v_{1}(\alpha)+\cdots+2(p-1) v_{p-1}(\alpha)$ which is the cohomological degree of the element $\alpha$ in QSymm $\left(Z_{(p)}\right)$.

The map $C P^{\infty} \longrightarrow B U$ corresponds to the $K$-theory orientation of $C P^{\infty}$ in $k u^{2}\left(C P^{\infty}\right)$. As $B U \simeq \Omega S U$, there is an adjoint map $\Sigma C P^{\infty} \longrightarrow S U$. Since the Adams splitting is compatible with the infinite loop space structure on $B U$, the delooping of $B U_{(p)}, \mathrm{B} B U_{(p)} \simeq S U_{(p)}$, splits into delooped pieces $\mathrm{B} W_{1} \times$ $\cdots \times \mathrm{B} W_{p-1}$. For a fixed $j$ in the range $1 \leqslant j \leqslant p-1$, the homology generators $\beta_{i} \in H_{2 i}\left(C P^{\infty} ; \mathrm{Z}_{(p)}\right)$ with $i \equiv j \bmod (p-1)$ stem from $H_{*}\left(Y_{j} ; \mathbf{Z}_{(p)}\right)$. The orientation maps $\beta_{i}$ to the $i$ th generator $b_{i}$ in $H_{*}\left(B U ; Z_{(p)}\right)$ and this lives on the corresponding Adams summand. 


\section{Steenrod operations on QSymm $\left(F_{p}\right)$}

Working with $\mathrm{F}_{p}$-coefficients we can ask how the Steenrod operations connect the generators in $\mathrm{QSymm}\left(\mathrm{F}_{p}\right)$. We first state an easy result about $p$ th powers, which is stated without proof in [16, (7.17)].

Lemma 4.1. The $p$ th power of an element $\left[a_{1}, \ldots, a_{r}\right] \in \operatorname{QSymm}\left(\mathrm{F}_{p}\right)$ is equal to $\left[p a_{1}, \ldots, p a_{r}\right]$, i.e., $\mathscr{P}^{a_{1}+\cdots+a_{r}}\left(\left[a_{1}, \ldots, a_{r}\right]\right)=\left[p a_{1}, \ldots, p a_{r}\right]$.

Proof. Recall that $\left[a_{1}, \ldots, a_{r}\right]$ is dual to the generator $Z_{a_{1}} \cdot \ldots \cdot Z_{a_{r}}$ with respect to the monomial basis in NSymm. Thus we have to determine the element which corresponds to $\left(\left(Z_{a_{1}} \cdot \ldots \cdot Z_{a_{r}}\right)^{*}\right)^{p}$.

Setting $Z(t)=\sum_{i} Z_{i} t^{i}$, we have $\Delta\left(Z_{m}\right)=\sum_{i} Z_{i} \otimes Z_{m-i}$, so $Z(t)$ is group-like. We obtain that the $p$-fold iterate $\Delta^{p}$ of the coproduct on a monomial $Z_{j_{1}} \cdot \ldots \cdot Z_{j_{n}}$ is captured in the series

$$
\Delta^{p}\left(Z\left(t_{1}\right) \cdot \ldots \cdot Z\left(t_{n}\right)\right)=\Delta^{p}\left(Z\left(t_{1}\right)\right) \cdot \ldots \cdot \Delta^{p}\left(Z\left(t_{n}\right)\right)
$$

which can be expressed as the $p$-fold product

$$
\left(Z\left(t_{1}\right) \otimes \ldots \otimes Z\left(t_{n}\right)\right) \cdot \ldots \cdot\left(Z\left(t_{1}\right) \otimes \ldots \otimes Z\left(t_{n}\right)\right)=\left(Z\left(t_{1}\right) \cdot \ldots \cdot Z\left(t_{n}\right)\right)^{\otimes p} .
$$

Thus

$$
\sum_{\left(j_{1}, \ldots, j_{n}\right)}\left\langle\left[a_{1}, \ldots, a_{r}\right]^{p}, Z_{j_{1}} \cdot \ldots \cdot Z_{j_{n}}\right\rangle t_{1}^{j_{1}} \cdot \ldots \cdot t_{r}^{j_{n}}=t_{1}^{p j_{1}} \cdot \ldots \cdot t_{r}^{p j_{r}},
$$

therefore $\left[a_{1}, \ldots, a_{r}\right]^{p}$ is dual to $Z_{p a_{1}} \cdot \ldots \cdot Z_{p a_{r}}$.

We will determine the Steenrod operations in QSymm by using the James splitting (1.1) and the isomorphism of Theorem 1.1: there is an isomorphism of modules over the Steenrod algebra

$$
\widetilde{H}^{*}\left(\Omega \Sigma C P^{\infty} ; \mathrm{F}_{p}\right) \cong \bigoplus_{n} \widetilde{H}^{*}\left(\left(\mathrm{C} P^{\infty}\right)^{(n)} ; \mathrm{F}_{p}\right) .
$$

Let $x_{(i)} \in H^{2}\left(C P_{(i)}^{\infty}\right)$ be $c_{1}\left(\eta_{i}\right)$, where $C P_{(i)}^{\infty}$ denotes the $i$-th copy of $C P^{\infty}$ in the product $\left(C P^{\infty}\right)^{\times n}$ and $\eta_{i}$ is the line bundle induced from $\eta$ over $C P^{\infty}$. As $\beta_{n}$ is dual to $c_{1}^{n}$, the elements $x_{(1)}^{a_{1}} \cdot \ldots \cdot x_{(n)}^{a_{n}}$ give an additive basis of $H^{*}\left(\Omega \Sigma C P^{\infty}\right)$ and they correspond to the generators $\alpha=\left[a_{1}, \ldots, a_{n}\right]$. Since the James splitting (1.1) is only defined after one suspension, we cannot read off the multiplicative structure of $H^{*}\left(\Omega \Sigma C P^{\infty}\right)$ immediately.

For two generators $\alpha$ and $\beta$ in QSymm we denote their concatenation by $\alpha * \beta$. Thus for $\alpha$ as above and $\beta=\left[b_{1}, \ldots, b_{m}\right]$, we have

$$
\alpha * \beta=\left[a_{1}, \ldots, a_{n}, b_{1}, \ldots, b_{m}\right] .
$$


The Cartan formula leads to a nice description of the action of the Steenrod algebra on QSymm $\left(\mathrm{F}_{p}\right)$.

Proposition 4.2. The following Cartan formula holds for the star product:

$$
\mathscr{P}^{i}(\alpha * \beta)=\sum_{k+\ell=i} \mathscr{P}^{k}(\alpha) * \mathscr{P}^{\ell}(\beta)
$$

Proof. Identifying $\alpha$ and $\beta$ with their corresponding elements in the cohomology of a suitable smash power of $C P^{\infty}$, the Cartan formula on $H^{*}\left(\left(C P^{\infty}\right)^{(n+m)} ; \mathrm{F}_{p}\right)$ becomes

$$
\begin{aligned}
& \mathscr{P}^{i}\left(x_{(1)}^{a_{1}} \cdot \ldots \cdot x_{(n)}^{a_{n}} x_{(n+1)}^{b_{1}} \cdot \ldots \cdot x_{(n+m)}^{b_{m}}\right) \\
& \quad=\sum_{k+\ell=i} \mathscr{P}^{k}\left(x_{(1)}^{a_{1}} \cdot \ldots \cdot x_{(n)}^{a_{n}}\right) \mathscr{P}^{\ell}\left(x_{(n+1)}^{b_{1}} \cdot \ldots \cdot x_{(n+m)}^{b_{m}}\right) .
\end{aligned}
$$

Identifying $x_{(n+i)}^{b_{i}}$ with $x_{(i)}^{b_{i}}$ gives the result.

As we can write every element $\alpha=\left[a_{1}, \ldots, a_{n}\right]$ as $\left[a_{1}\right] * \ldots *\left[a_{n}\right]$ it suffices to describe the Steenrod operations on the elements of the form $[n]$ with $n \in \mathrm{N}$. The following is easy to verify using standard identities for binomial coefficients mod $p$ and from Lemma 4.1. We recall that when $s>r$, $\left(\begin{array}{l}r \\ s\end{array}\right)=0$.

Proposition 4.3. If the $p$-adic expansions of $n$ and $k$ are $n_{0}+n_{1} p+\ldots+$ $n_{r} p^{r}$ and $k_{0}+k_{1} p+\ldots+k_{s} p^{s}$ respectively, where $s \leqslant r$, then

$$
\mathscr{P}^{k}[n]=\left(\begin{array}{l}
n \\
k
\end{array}\right)[n+k(p-1)]=\left(\begin{array}{l}
n_{0} \\
k_{0}
\end{array}\right) \ldots\left(\begin{array}{l}
n_{s} \\
k_{s}
\end{array}\right)[n+k(p-1)] .
$$

Hence if $p \nmid\left(k_{0}-n_{0}\right)$ and if $n_{i} \geqslant k_{i}$ for all $1 \leqslant i \leqslant s$, then $\mathscr{P}^{k}[n]$ is a non-zero indecomposable. When $p \nmid\left(k_{0}-n_{0}\right)$ but $n_{i}<k_{i}$ for some $i$, the right hand side is zero, and for $p \mid\left(k_{0}-n_{0}\right)$ the right hand side is decomposable.

For example, the power operations $\mathscr{P}^{k}$ on the elements $\alpha=\left[a_{1}, \ldots, a_{n}\right]$ with $1 \leqslant a_{i} \leqslant p-1$ and $k \leqslant \operatorname{deg}(\alpha)$ yield non-trivial sums of indecomposables.

\section{Witt vectors and the cohomology of $B U$}

The self-dual bicommutative Hopf algebras $H_{*}(B U) \cong H^{*}(B U)$ are closely related to both lambda rings and Witt vectors (see [15]). In particular, there are $p$-local splittings due to Husemöller [20], subsequently refined to take into 
account the Steenrod actions in [4]. Here is a brief account of this theory over any commutative ring $R$.

First consider the graded commutative Hopf algebra

$$
H_{*}(B U ; R)=R\left[b_{n} \mid n \geqslant 1\right]
$$

where $b_{n} \in H_{2 n}(B U ; R)$ is the image of the canonical generator of $H_{2 n}\left(\mathrm{C} P^{\infty} ; R\right)$ as defined in [1], and the coproduct is determined by

$$
\Delta\left(b_{n}\right)=\sum_{i+j=n} b_{i} \otimes b_{j}
$$

This coproduct makes the formal power series

$$
b(t):=\sum_{n \geqslant 0} b_{n} t^{n} \in H_{*}(B U)[[t]]
$$

grouplike, i.e.,

$$
\Delta b(t)=b(t) \otimes b(t),
$$

or equivalently

$$
\sum_{n \geqslant 0} \Delta\left(b_{n}\right) t^{n}=\left(\sum_{n \geqslant 0} b_{m} t^{m}\right) \otimes\left(\sum_{n \geqslant 0} b_{n} t^{n}\right) .
$$

There is an obvious isomorphism of graded Hopf algebras over $R$,

$$
H_{*}(B U ; R) \cong \operatorname{Symm}(R)
$$

under which $b_{n} \leftrightarrow c_{n}$.

For each $n \geqslant 1$, there is a cyclic primitive submodule

$$
\operatorname{Prim}(\operatorname{Symm}(R))_{2 n}=R\left\{q_{n}\right\} \text {, }
$$

where the generators are defined recursively by $q_{1}=b_{1}$ together with the Newton formula

$$
q_{n}=b_{1} q_{n-1}-b_{2} q_{n-2}+\cdots+(-1)^{n} b_{n-1} q_{1}+(-1)^{n-1} n b_{n} .
$$

The $q_{n}$ can be defined using generating functions and logarithmic derivatives as follows.

$$
\sum_{n \geqslant 1}(-1)^{n-1} q_{n} t^{n-1}=\frac{d}{d t} \log (b(t))=\frac{b^{\prime}(t)}{b(t)} .
$$


The recursion (5.1) then follows via multiplication by $b(t)$. The fact that the $q_{n}$ are primitive follows because the logarithmic derivative maps products to sums.

Now we introduce another family of elements $v_{n} \in \operatorname{Symm}(R)_{2 n}$ which are defined by generating functions using an indexing variable $t$ through the formula

$$
\prod_{k \geqslant 1}\left(1-v_{k} t^{k}\right)=\sum_{n \geqslant 0} b_{n}(-t)^{n}
$$

These also satisfy

$$
q_{n}=\sum_{k \ell=n} k v_{k}^{\ell}
$$

THEOREM 5.1. The elements $v_{n} \in \operatorname{Symm}(R)_{2 n}$ are polynomial generators for $\operatorname{Symm}(R)$,

$$
\operatorname{Symm}(R)=R\left[v_{n} \mid n \geqslant 1\right] .
$$

The coproduct is given by the recursion

$$
\sum_{k \ell=n} k \Delta\left(v_{k}\right)^{\ell}=\sum_{k \ell=n} k\left(v_{k}^{\ell} \otimes 1+1 \otimes v_{k}^{\ell}\right)
$$

When $R$ is a $\mathbf{Z}_{(p)}$-algebra, for each $n$ such that $p \nmid n$, there are elements $v_{n, r} \in \operatorname{Symm}(R)_{2 n p^{r}}$ defined recursively by

$$
q_{n p^{r}}=p^{r} v_{n, r}+p^{r-1} v_{n, r-1}^{p}+\cdots+v_{n, 0}^{p^{r}} .
$$

Then the subalgebra

$$
R\left[v_{n, r} \mid r \geqslant 0\right] \subseteq \operatorname{Symm}(R)
$$

is a sub Hopf algebra. The following result was first introduced into topology in [20].

Theorem 5.2. If $R$ is a $\mathbf{Z}_{(p)}$-algebra, there is a decomposition of Hopf algebras

$$
\operatorname{Symm}(R)=\bigotimes_{p \nmid n} R\left[v_{n, r} \mid r \geqslant 0\right] .
$$

Notice that when $R$ is an $\mathrm{F}_{p}$-algebra, we have $q_{n p^{r}}=q_{n}^{p^{r}}$.

There are Frobenius and Verschiebung Hopf algebra endomorphisms

$$
\mathbf{f}_{d}, \mathbf{v}_{d}: \operatorname{Symm}(R) \longrightarrow \operatorname{Symm}(R)
$$


given by

$$
\mathbf{f}_{d}\left(v_{n}\right)=v_{n d}, \quad \mathbf{v}_{d}\left(v_{n}\right)= \begin{cases}d v_{n / d} & \text { if } d \mid n, \\ 0 & \text { otherwise. }\end{cases}
$$

In the dual $H^{*}(B U ; R)$, we have the universal Chern classes $c_{i}$ and the primitives $s_{i}$ and under there is an isomorphism of Hopf algebras

$$
H_{*}(B U ; R) \stackrel{\cong}{\rightrightarrows} H^{*}(B U ; R) ; \quad b_{i} \leftrightarrow c_{i}, \quad q_{i} \leftrightarrow s_{i} .
$$

We define the element $w_{i}$ to be the image of the element $v_{i}$ under this isomorphism. When localized at a prime $p$, we define $w_{n, r}$ to be the element corresponding to $v_{n, r}$. The coproduct on the $w_{i}$ is computed using the analogue of Equation (5.3),

$$
\prod_{k \geqslant 1}\left(1-w_{k} t^{k}\right)=\sum_{n \geqslant 0} c_{n}(-t)^{n}
$$

together with the Cartan formula for the $c_{i}$.

The $c_{i}$ can be identified with elementary symmetric functions in infinitely many variables, say $x_{i}$, and the coproduct $\psi_{\oplus}$ on a symmetric function $f\left(x_{i}\right)$ amounts to splitting the variables into two infinite collections, say $x_{i}^{\prime}, x_{i}^{\prime \prime}$, and expressing the symmetric function $f\left(x_{i}^{\prime}, x_{j}^{\prime \prime}\right)$ in terms of symmetric functions of these subsets. There is a second coproduct $\psi_{\otimes}$ corresponding to replacing $f\left(x_{i}\right)$ by $f\left(x_{i}^{\prime}+x_{j}^{\prime \prime}\right)$. There is an interpretation of this structure in terms of symmetric functions. For example, the latter coproduct gives

$$
\psi_{\otimes}\left(s_{n}\right)=\sum_{0 \leqslant i \leqslant n}\left(\begin{array}{l}
n \\
i
\end{array}\right) s_{i} \otimes s_{n-i} .
$$

Both of these coproducts are induced by topological constructions. Let us recall that the space $B U$ admits maps that represent the Whitney sum and tensor products of bundles,

$$
B U \times B U \stackrel{\oplus}{\rightarrow} B U, \quad B U \times B U \stackrel{\otimes}{\rightarrow} B U
$$

Using the Splitting Principle, it is standard that the resulting coproducts

$$
H^{*}(B U) \longrightarrow H^{*}(B U) \otimes H^{*}(B U)
$$

induced by these are equal to our two coproducts $\psi_{\oplus}, \psi_{\otimes}$.

Let $R$ be again a commutative ring with unit. There are two endofunctors $\Lambda(-)$ and $W(-)$ on the category of commutative rings, Rings. Details of this can be found in [15, chapter III], in particular in E.2, although the construction 
there is of an inhomogeneous version corresponding to $K^{0}(B U)$ rather than $H^{*}(B U)$. We will first describe their values in the category of sets.

DeFinition 5.3. Let $\Lambda(R)$ be $1+t R[[t]]$ with addition given by the multiplication of power series and let the (big) Witt vectors on $R, W(R)$, be the product $\prod_{i \geqslant 1} R$.

Consider the following representable functor Rings(Symm, - ). The coproducts $\psi_{\oplus}$ and $\psi_{\otimes}$ make this into a ring scheme. Therefore they induce two different commutative multiplications on $\operatorname{Rings}($ Symm, $R)$.

Let $\varphi_{\Lambda}: \operatorname{Rings}($ Symm,$R) \longrightarrow \Lambda(R)$ be the bijection that sends a map $f$ from Symm to $R$ to the power series

$$
1+f\left(c_{1}\right)(-t)+f\left(c_{2}\right)(-t)^{2}+\cdots+f\left(c_{n}\right)(-t)^{n}+\cdots .
$$

The coproduct $\psi_{\oplus}$ corresponds to the multiplication of power series which should be thought of as a kind of addition, whereas $\psi_{\otimes}$ gives a multiplication. These two operations interact to make this a functor with values in commutative rings.

For the Witt vectors, $W(R)$, take the bijection $\varphi_{W}$ : $\operatorname{Rings}(\operatorname{Symm}, R) \longrightarrow$ $W(R)$ that sends $f$ to the sequence $\left(f\left(w_{i}\right)\right)_{i \geqslant 1}$ where the $w_{i}$ are given as in (5.5). Therefore, the two coproducts $\psi_{\oplus}$ and $\psi_{\otimes}$ induce a ring structure on $W(R)$.

THEOREM 5.4. The two different $H$-space structures, $B U_{\oplus}$ and $B U_{\otimes}$ give rise to two comultiplications on Symm via $\psi_{\oplus}$ and $\psi_{\otimes}$ which together induce a ring structure on the big Witt vector $W(R)$ ring. This ring structure coincides with the standard one as it is described for instance in [15, III §17].

Proof. The exponential map $[15,(17.2 .7)]$ sends a Witt vector $\left(a_{1}, a_{2}, \ldots\right)$ $\in W(R)$ to the element $\prod_{i \geqslant 1}\left(1-a_{i} t^{i}\right) \in \Lambda(R)$. Formula (5.5) ensures that this isomorphism of rings sends the sequence of generators $\left(w_{n}\right)_{n}$ to the product $\prod_{i \geqslant 1}\left(1+(-1)^{i} c_{i} t^{i}\right)$. That the ring structure agrees for $\Lambda(R)$ follows directly from the definition.

\section{Quasi-Witt vectors}

In the case of symmetric functions, we interpreted the addition and multiplication of Witt vectors as coming from the two $H$-space structures on $B U$. On $\Omega \Sigma C P^{\infty}$ we have the ordinary $H$-space structure, $\mu_{\oplus}: \Omega \Sigma C P^{\infty} \times$ $\Omega \Sigma C P^{\infty} \longrightarrow \Omega \Sigma C P^{\infty}$, coming from loop addition. In addition to this, we consider the following construction. Essentially the same construction already appears in [26], the difference arises from various choices concerning the Hopf construction. 
Let $X$ be an $H$-space. For two loops $f, g: S^{1} \longrightarrow \Sigma X$, their product $f \diamond g$ is the composition

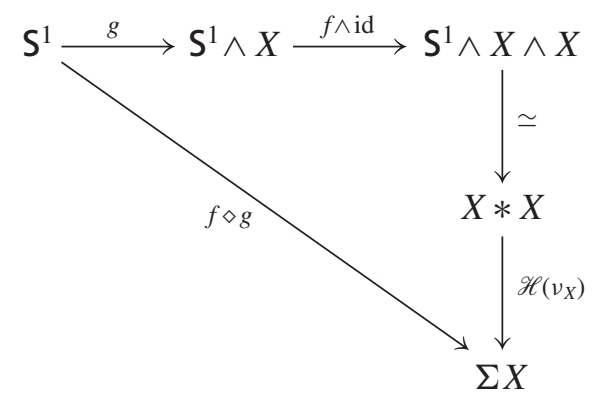

in which the last map is the Hopf construction $\mathscr{H}$ on the $H$-space multiplication $v_{X}$ on $X$ from the reduced join of two copies of $X$, see [34, XI, §4].

LEMMA 6.1. The $\diamond$-product defines a map $\diamond: \Omega \Sigma X \wedge \Omega \Sigma X \longrightarrow \Omega \Sigma X$. It satisfies the following left distributivity law for loops $f, g, h \in \Omega \Sigma X$ :

$$
f \diamond\left(\mu_{\oplus}(g, h)\right) \simeq \mu_{\oplus}(f \diamond g, f \diamond h) .
$$

If $i: X \longrightarrow \Omega \Sigma X$ is the natural map, then for any $x \in X$ the following right distributivity law holds:

$$
\mu_{\oplus}(g, h) \diamond i(x) \simeq \mu_{\oplus}(g \diamond i(x), h \diamond i(x)) .
$$

A map of $H$-spaces $\ell: X \longrightarrow Y$ is compatible with the $\diamond$-operations in that it satisfies

$$
\Omega \Sigma(\ell) \diamond \Omega \Sigma(\ell) \simeq \Omega \Sigma(\ell) \circ \diamond .
$$

Proof. It is obvious that the $\diamond$-product with the constant loop on either side gives the constant loop again. For the distributivity law we consider the following diagram

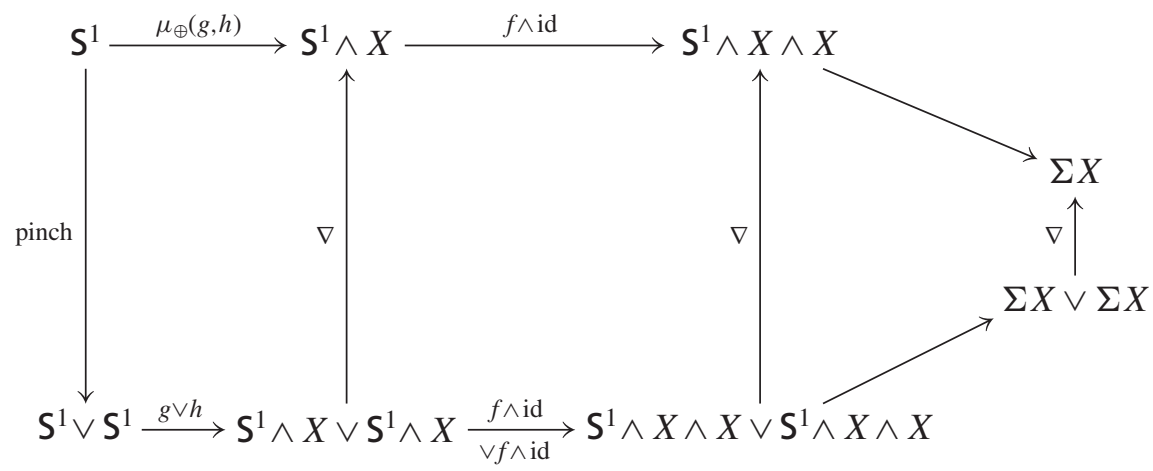


in which all squares commute and the upper composition corresponds to $f \diamond$ $\left(\mu_{\oplus}\right)$ whereas the lower composition is $\mu_{\oplus}(f \diamond g, f \diamond h)$. Hence the diagram commutes and left distributivity is verified.

A simpler argument gives the right distributivity for loops of the form $i(x)$. Naturality of the diamond product with respect to maps of $H$-spaces follows because the Hopf-construction $\mathscr{H}$ on an $H$-space multiplication $v$ satisfies

$$
\mathscr{H}\left(v_{Y}\right) \circ(\ell * \ell) \sim \Sigma(\ell) \circ \mathscr{H}\left(v_{X}\right)
$$

for $\ell$ an $H$-map from $X$ to $Y$ [34, XI.4].

For the following, we recall the notion of a near-ring, see [27] for example. Briefly, a near-ring is a group equipped with a second product which left or right distributes over the group operation and is associative. Various extra conditions are sometimes imposed such as the existence of a multiplicative unit. Dropping the associativity requirement leads to the notion of a quasi-near-ring which we refrain from using!

REMARK 6.2. The structure $\left(\Omega \Sigma X, \mu_{\oplus}, \diamond\right)$ for an associative $H$-space $X$ from Lemma 6.1 might be called a left (non-unital, non-associative) near-ring in the homotopy category and we abbreviate that to homotopy near-ring. By functoriality, $H_{*}\left(\Omega \Sigma C P^{\infty}\right) \cong$ NSymm inherits such a structure in the category of graded Z-coalgebras. Dually, $H^{*}\left(\Omega \Sigma C P^{\infty}\right) \cong$ QSymm is a co-near-ring in the category of graded commutative rings.

DeFinition 6.3. Let $R$ be a commutative ring with unit. We call

$$
Q W(R):=\operatorname{Rings}(\text { QSymm, } R)
$$

the near-ring of quasi-Witt vectors on $R$.

Note that these quasi-Witt vectors, $Q W(R)$ do not agree with Hazewinkel's noncommutative Witt vectors $M(R)$ of [17, p. 75]. Our coproducts are induced by maps on spaces, hence they are homogeneous. Hazewinkel's formula [17, $\S 12]$ gives a non-homogeneous coproduct. They might agree if we used a $K$-theoretic analogue in place of our homological one.

From Selick [30, p. 84] we can deduce an explicit formula for the $\diamond$ product on basic loops $i(x): t \mapsto[t, x] \in \Sigma X$ for an associative $H$-space $X$. It will send two of such basic loops $i(x)$ and $i(y)$ to $\mu_{\oplus}(\overline{i(y)}, i(x) \cdot i(y), \overline{i(x)})$, where . is the $H$-product on $X$ and ( ) denotes loop reversal. This allows us to calculate $Z_{i} \diamond Z_{j}$ in $H_{*}\left(\Omega \Sigma C P^{\infty}\right)$. We note that by construction the diamond product is really defined on reduced homology. When $x$ is a positive degree element of $H_{*}\left(\Omega \Sigma C P^{\infty}\right)$, we have

$$
1 \diamond x=0=x \diamond 1
$$


Recall that $Z(t)=\sum_{i \geqslant 0} Z_{i} t^{i}$.

Proposition 6.4. The diamond product of the two generators $Z_{i}, Z_{j}$ is

$$
Z_{i} \diamond Z_{j}=\sum_{r=0}^{i} \sum_{s=0}^{j}\left(\begin{array}{c}
r+s \\
r
\end{array}\right) \chi\left(Z_{j-s}\right) Z_{r+s} \chi\left(Z_{i-r}\right) .
$$

In terms of generating functions this is equivalent to

$$
Z(s) \diamond Z(t)=Z(t)^{-1} Z(s+t) Z(s)^{-1} .
$$

Proof. Note that in order to apply Selick's formula, we have to use the diagonal

$$
\Omega \Sigma C P^{\infty} \times \Omega \Sigma C P^{\infty} \longrightarrow\left(\Omega \Sigma C P^{\infty}\right)^{4} .
$$

On homology this corresponds to taking the coproduct of $Z_{i}$ and $Z_{j}$, thus $Z_{i} \otimes Z_{j}$ maps to

$$
\sum_{r=0}^{i} \sum_{s=0}^{j} Z_{r} \otimes Z_{i-r} \otimes Z_{s} \otimes Z_{j-s} .
$$

We have to switch factors and apply the $C P^{\infty}-H$-multiplication to $Z_{r} \otimes Z_{s}$ to give the term $\left(\begin{array}{c}r+s \\ r\end{array}\right) Z_{r+s}$ because the cohomology of $C P^{\infty}$ is primitively generated. We now apply the loop-inversion antipode in $H_{*}\left(\Omega \Sigma C P^{\infty}\right)$ to the remaining factors, then finally, we use loop multiplications to obtain the result.

We can calculate products of the form $u \diamond\left(v_{1} \ldots v_{n}\right)$ with $u, v_{1}, \ldots, v_{n}$ all of positive degree by using left distributivity (6.1) and (6.3). Here we denote the loop product by juxtaposing homology elements, i.e., $x y=x \cdot y$. For example, if the coproduct on $u$ is

$$
\Delta(u)=u \otimes 1+1 \otimes u+\sum_{r} u_{r}^{\prime} \otimes u_{r}^{\prime \prime},
$$

so that $\sum_{r} u_{r}^{\prime} \otimes u_{r}^{\prime \prime}$ is the reduced coproduct of $u$, then we have

$$
u \diamond\left(v_{1} v_{2}\right)=\sum_{r}\left(u_{r}^{\prime} \diamond v_{1}\right)\left(u_{r}^{\prime \prime} \diamond v_{2}\right)
$$

In particular, for positive degree elements $x, y$, this gives

$$
Z_{i} \diamond(x y)=\sum_{r=1}^{i-1}\left(Z_{r} \diamond x\right)\left(Z_{i-r} \diamond y\right)
$$


for any loop product $x y$ of positive degree elements in $H_{*}\left(\Omega \Sigma C P^{\infty}\right)$. Similarly, right distributivity (6.2) gives

$$
(x y) \diamond Z_{i}=\sum_{r=1}^{i-1}\left(x \diamond Z_{r}\right)\left(y \diamond Z_{i-r}\right) .
$$

In particular,

$$
\begin{aligned}
& Z_{1} \diamond(x y)=0, \\
& (x y) \diamond Z_{1}=0 .
\end{aligned}
$$

The first of these is a special case of the more general

Lemma 6.5. If $u$ is primitive, then for all positive degree elements $x, y$,

$$
u \diamond(x y)=0,
$$

i.e., the left diamond product with a primitive annihilates decomposables.

Proof. This follows immediately from Equations (6.3) and (6.6).

In the non-commutative algebra NSymm there are two natural families of primitives analogous to the family $\left(q_{n}\right)_{n \in \mathrm{N}}$ from (5.1). We define

$$
\begin{aligned}
& \sum_{n \geqslant 1}(-1)^{n-1} Q_{n} t^{n-1}=Z(t)^{-1} Z^{\prime}(t), \\
& \sum_{n \geqslant 1}(-1)^{n-1} Q_{n}^{\prime} t^{n-1}=Z^{\prime}(t) Z(t)^{-1},
\end{aligned}
$$

where $Z^{\prime}(t)$ is the derivative of the series $Z(t)$. These two generating functions are related through conjugation by the $Z(t)$-series. Multiplication by $Z(t)$ on the left (resp. right) gives the recursion formulae

$$
\begin{aligned}
& \text { (6.13) } Q_{n}=Z_{1} Q_{n-1}-Z_{2} Q_{n-2}+\cdots+(-1)^{n} Z_{n-1} Q_{1}+(-1)^{n-1} n Z_{n}, \\
& \text { (6.14) } Q_{n}^{\prime}=Q_{n-1}^{\prime} Z_{1}-Q_{n-2}^{\prime} Z_{2}+\cdots+(-1)^{n} Q_{1}^{\prime} Z_{n-1}+(-1)^{n-1} n Z_{n}
\end{aligned}
$$

These families agree with Hazewinkel's [18, pp. 328-329] up to sign. As the antipode on the $Z_{i}$ is given by the generating function

$$
\sum_{i \geqslant 0} \chi\left(Z_{i}\right) t^{i}=Z(t)^{-1}
$$


we also obtain the formulae

$$
\begin{aligned}
Q_{n} & =(-1)^{n-1} \sum_{j=1}^{n} j \chi\left(Z_{n-j}\right) Z_{j}, \\
Q_{n}^{\prime} & =(-1)^{n-1} \sum_{j=1}^{n} j Z_{j} \chi\left(Z_{n-j}\right) .
\end{aligned}
$$

Proposition 6.6. The primitives $Q_{n}$ and $Q_{n}^{\prime}$ can be expressed as

$$
Q_{n}=(-1)^{n-1} Z_{1} \diamond Z_{n-1}, \quad Q_{n}^{\prime}=(-1)^{n-1} Z_{n-1} \diamond Z_{1} .
$$

Proof. Using (6.5) and taking the derivative with respect to $s$ at $s=0$ we obtain

$$
Z_{1} \diamond Z(t)=Z(t)^{-1} Z^{\prime}(t)-Z_{1},
$$

which gives

$$
Z_{1} \diamond Z_{n-1}=(-1)^{n-1} Q_{n} .
$$

Similarly, using the derivative with respect to $t$ at $t=0$ gives

$$
Z(s) \diamond Z_{1}=Z^{\prime}(s) Z(s)^{-1}-Z_{1}
$$

and we get the corresponding formula for $Q_{n}^{\prime}$.

We can calculate the recursively defined 'power'

$$
Z_{1}^{\diamond n}=Z_{1} \diamond\left(Z_{1}^{\diamond(n-1)}\right),
$$

which is actually a spherical element since $Z_{1}$ is spherical. An easy induction on $n$ yields

$$
Z_{1}^{\diamond n}=(-1)^{n-1}(n-1) ! Q_{n} .
$$

Related formulae given in terms of (6.15) occur in [26, corollary 5.2] and further spherical primitives are also determined there. We could also replace the above recursion by the one involving $\left(Z_{1}^{\diamond(n-1)}\right) \diamond Z_{1}$ which yields $(-1)^{n-1}(n-$ $1) ! Q_{n}^{\prime}$ in place of $(-1)^{n-1}(n-1) ! Q_{n}$.

Now we give a modification of the above construction. For us, a spectrum will mean a collection of spaces $E=\left\{E_{n}\right\}$ and suitably related homeomorphisms $\sigma_{n, 1}: E_{n} \longrightarrow \Omega E_{n+1}$ with adjoint evaluation maps $\widetilde{\sigma}_{n, 1}: \Sigma E_{n} \longrightarrow E_{n+1}$. We obtain maps $\sigma_{n, m}: E_{n} \longrightarrow \Omega^{m} E_{n+m}$ as iterations of the maps $\sigma_{n, 1}$ and we denote the inverse of $\sigma_{n, m}$ by $\phi_{n+m, n}$.

Such structure is known to be present for many important ring spectra such as those of complex $K$-theory which is our main concern, it is always present 
when $E$ is an $E_{\infty}$ ring spectrum. We also write $E^{\prime}=\left\{E_{n}^{\prime}\right\}$ for the 0 -connected cover of $E$, so $E_{0}^{\prime}$ is the connected component of the basepoint in $E_{0}$.

Let $U_{1}(E)$ denote the component of $E_{0}$ corresponding to 1 in the ring $\pi_{0}(E)=\pi_{0}\left(E_{0}\right)$. Then $U_{1}(E)$ admits the structure of an $H$-space under the restriction of $\mu_{0,0}: E_{0} \wedge E_{0} \longrightarrow E_{0}$, and we denote this multiplication by $\mu$.

There is a homotopy equivalence $\lambda: U_{1}(E) \longrightarrow E_{0}^{\prime}$ which satisfies

$$
\lambda \circ \mu \simeq(\mu \circ \lambda \times \lambda) *\left(\lambda \circ \mathrm{pr}_{1}\right) *\left(\lambda \circ \mathrm{pr}_{2}\right),
$$

where $*$ denotes the sum in $E_{0}$ (loop product) and $\mathrm{pr}_{1}, \mathrm{pr}_{2}$ are the two projections from $U_{1}(E) \times U_{1}(E)$. This is defined by shifting component by adding an element in the (-1)-component of $E_{0}$. In terms of the cohomology ring $E^{0}(X)$ for a connected space $X$, this says that the associated natural transformation

$$
\bar{\lambda}: U_{1}(E)^{0}(X)=1+\widetilde{E}^{0}(X) \longrightarrow \widetilde{E}^{0}(X)
$$

satisfies

$$
\bar{\lambda}(x y)=\bar{\lambda}(x) \bar{\lambda}(y)+\bar{\lambda}(x)+\bar{\lambda}(y) .
$$

We can endow $E_{0}^{\prime}$ with the $H$-structure under which $\lambda$ is an $H$-space equivalence. Then we obtain an induced equivalence of homotopy near-rings

$$
\Omega \Sigma(\lambda): \Omega \Sigma U_{1}(E) \longrightarrow \Omega \Sigma E_{0}^{\prime} .
$$

The evaluation map $\widetilde{\sigma}_{0,1}: \Sigma E_{0}^{\prime} \longrightarrow E_{1}^{\prime}$ induces a loop map

$$
\theta_{0}:=\phi_{1,0} \circ \Omega \widetilde{\sigma}_{0,1}: \Omega \Sigma E_{0}^{\prime} \longrightarrow E_{0}^{\prime},
$$

where $E_{0}^{\prime}$ forms a homotopy ring object with loop addition and $\mu^{\prime}: E_{0}^{\prime} \wedge E_{0}^{\prime} \longrightarrow$ $E_{0}^{\prime}$, the restriction of the multiplication to the 0-component of $E_{0}$.

TheOREM 6.7. The map $\theta_{0}: \Omega \Sigma E_{0}^{\prime} \longrightarrow E_{0}^{\prime}$ induces a homotopy commutative diagram of loop spaces

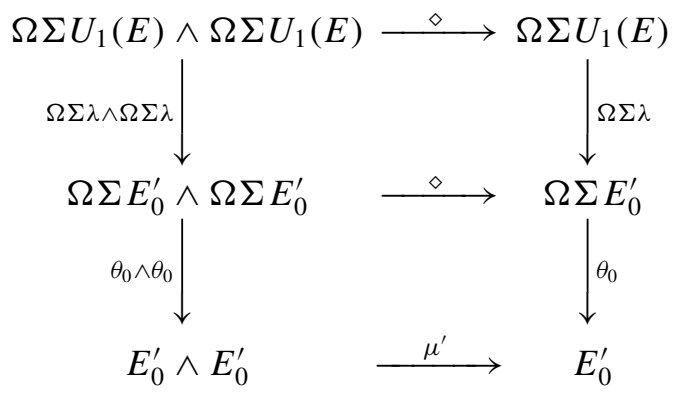

and hence $\theta_{0}$ and the composition $\theta_{0} \circ \Omega \Sigma(\lambda)$ induce maps of homotopy nearrings. 
Proof. We already know that the top square commutes. For the lower square we consider the following diagram.

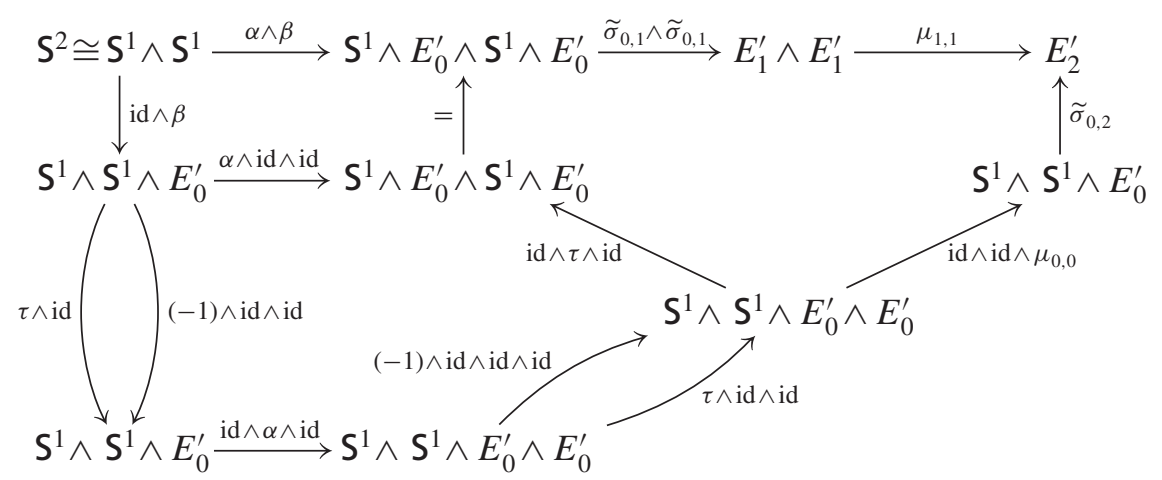

As we have that

$$
(\operatorname{id} \wedge \tau \wedge \mathrm{id}) \circ(\tau \wedge \mathrm{id} \wedge \mathrm{id}) \circ(\mathrm{id} \wedge \alpha \wedge \mathrm{id}) \circ(\tau \wedge \mathrm{id})=\alpha \wedge \mathrm{id} \wedge \mathrm{id},
$$

the lower left outer pentagon commutes. The maps $\tau \wedge \mathrm{id} \wedge \mathrm{id}$ and $(-1) \wedge$ $\mathrm{id} \wedge \mathrm{id} \wedge \mathrm{id}$ are homotopic thus the lower left inner pentagon commutes up to homotopy. The top horizontal map is the smash product of $\alpha$ and $\beta$ followed by evaluation and multiplication. The upper left square commutes and the upper right pentagon commutes up to homotopy. Therefore this composite is homotopic to

$$
\begin{aligned}
\widetilde{\sigma}_{0,2} \circ\left(\mathrm{id} \wedge \mathrm{id} \wedge \mu_{0,0}\right) \circ((-1) & \wedge \mathrm{id} \wedge \mathrm{id} \wedge \mathrm{id}) \\
\circ(\mathrm{id} \wedge \alpha & \wedge \mathrm{id}) \circ((-1) \wedge \mathrm{id} \wedge \mathrm{id}) \circ(\mathrm{id} \wedge \beta)
\end{aligned}
$$

and this composite is $\widetilde{\sigma}_{0,2} \circ \Sigma(\alpha \diamond \beta)$.

Corollary 6.8. Let $R$ be a commutative ring for which $H_{*}\left(E_{0}^{\prime} ; R\right)$ is $R$-flat. Then the maps $\theta_{0}$ and $\theta_{0} \circ \Omega \Sigma(\lambda)$ induce epimorphisms of near-ring objects in $R$-coalgebras

$$
H_{*}\left(\Omega \Sigma E_{0}^{\prime} ; R\right) \longrightarrow H_{*}\left(E_{0}^{\prime} ; R\right) \longleftarrow H_{*}\left(\Omega \Sigma U_{1}(E) ; R\right) .
$$

Proof. This follows immediately from the fact that the inclusion map $E_{0}^{\prime} \longrightarrow \Omega \Sigma E_{0}^{\prime}$ is a right homotopy inverse for $\theta_{0}$.

Returning to our main example, we take $E=k u$ and $E^{\prime}=\Sigma^{2} k u$, thus $E_{0}=B U \times \mathrm{Z}$ and $E_{0}^{\prime}=B U$. Notice that we have a map of $H$-spaces 
$\nu: C P^{\infty} \longrightarrow U_{1}(K U)=B U_{\otimes}$ classifying line bundles. Our main conclusion is contained in

Theorem 6.9. The map $\Theta=\theta_{0} \circ \Omega \Sigma(\lambda \circ v): C P^{\infty} \longrightarrow B U$ induces $a$ commutative diagram

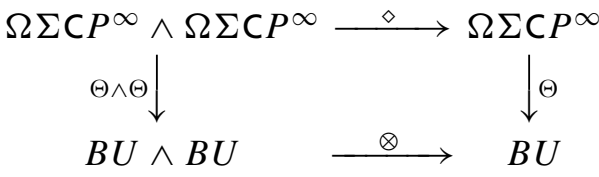

and hence $\Theta$ induces a map of homotopy near-rings. Furthermore, $\Theta$ induces an epimorphism $\Theta_{*}: H_{*}\left(\Omega \Sigma C P^{\infty}\right) \longrightarrow H_{*}(B U)$ of near-ring objects in Zcoalgebras.

Of course, $H_{*}(B U)$ is already a (non-unital) ring object in Z-coalgebras.

\section{The Thom spectrum of $\xi$}

The map $j: \Omega \Sigma C P^{\infty} \longrightarrow B U$ is a loop map and hence the Thom spectrum $M \xi$ of the virtual bundle $\xi$ is an $A_{\infty}$ ring spectrum and the natural map $M \xi \longrightarrow$ $M U$ is one of $A_{\infty}$ ring spectra, or equivalently of S-algebras in the sense of [11]. Since the homology $H_{*}(M \xi)$ is isomorphic as a ring to $H_{*}\left(\Omega \Sigma C P^{\infty}\right)$, we see that $M \xi$ is not even homotopy commutative, let alone an $E_{\infty}$ ring spectrum or equivalently a commutative S-algebra.

Let $\mathscr{A}_{p}^{*}$ be the mod $p$ Steenrod algebra and let $\mathscr{A}_{p}^{*} /\left(Q^{0}\right)$ be the quotient by the two-sided ideal generated by the Bockstein $Q^{0}$.

We also have in all cases, $H^{*}\left(B P ; \mathrm{F}_{p}\right) \cong \mathscr{A}_{p}^{*} /\left(Q^{0}\right)$ as $\mathscr{A}_{p}^{*}$-modules, where $B P$ is the $p$-primary Brown-Peterson spectrum [2, II §16]. More generally, any $\mathscr{A}_{p}^{*}$-module on which $Q^{0}$ acts trivially can be viewed as a $\mathscr{A}_{p}^{*} /\left(Q^{0}\right)$ module. In particular, $H^{*}\left(M U ; \mathrm{F}_{p}\right)$ becomes a free $\mathscr{A}_{p}^{*} /\left(Q^{0}\right)$-module in this way, and therefore $M U_{(p)}$ splits as a wedge of suspensions of $B P$ 's. The proof of this uses a result of Milnor and Moore [24, theorem 4.4] which only requires the existence of an associative coalgebra structure. Although $M \xi$ is not a commutative ring spectrum we may still apply such an argument to its cohomology.

Proposition 7.1. Let $p$ be a prime. Then $H^{*}\left(M \xi ; \mathrm{F}_{p}\right)$ is a free $\mathscr{A}_{p}^{*} /\left(Q^{0}\right)$ module and hence $M \xi_{(p)}$ is a wedge of $B P$ 's.

Proof. The standard Milnor-Moore argument works since $M \xi$ is a ring spectrum and the map $M j: M \xi \longrightarrow M U$ induced from $j$ gives rise to a monomorphism $M j: H^{*}\left(M U ; \mathrm{F}_{p}\right) \longrightarrow H^{*}\left(M \xi ; \mathrm{F}_{p}\right)$. From this we can deduce that the copy of $\mathscr{A}_{p}^{*} /\left(Q^{0}\right)$ on the stable Thom class of $H^{*}\left(M U ; \mathrm{F}_{p}\right)$ 
maps isomorphically to one in $H^{*}\left(M \xi ; \mathrm{F}_{p}\right)$. Obstruction theory now leads to the existence of an equivalence $M \xi_{(p)} \longrightarrow \bigvee_{\lambda} \Sigma^{2 k_{\lambda}} B P$.

Of course, as described in [2], Quillen gave a multiplicative idempotent on $M U_{(p)}$ leading to a precise description of such a splitting into $B P$ 's. We expect that $M \xi$ has an adequate analogue of the universality for complex orientations possessed by $M U$ and required for Quillen's approach. We will investigate this in future work. We also remark that although the spectrum $M \xi$ appears to be related to that studied in [3], the latter is not a ring spectrum.

Instead, we note some algebraic facts that are useful here. Fixing a prime $p$, we set $\mathscr{A}^{*}=\mathscr{A}_{p}^{*}$ and write $\mathscr{A}_{*}$ for the dual Steenrod algebra. The dual of $\mathscr{A}_{p}^{*} /\left(Q^{0}\right)$ is the polynomial sub-Hopf algebra $\mathrm{F}_{p}\left[\zeta_{i} \mid i \geqslant 1\right] \subseteq \mathscr{A}_{*}$ generated by the conjugates $\zeta_{i}$ of the Milnor generators $\xi_{i}$ and there is an isomorphism of $\mathscr{A}_{*}$-comodule algebras

$$
H_{*}\left(B P ; \mathrm{F}_{p}\right) \cong \mathrm{F}_{p}\left[\zeta_{i} \mid i \geqslant 1\right] .
$$

There is also an isomorphism of $\mathscr{A}_{*}$-comodules

$$
H_{*}\left(M \xi ; \mathrm{F}_{p}\right) \cong \bigoplus_{\lambda} \Sigma^{2 k_{\lambda}} \mathrm{F}_{p}\left[\zeta_{i} \mid i \geqslant 1\right] .
$$

For any commutative ring $R$, under the Thom isomorphism

$$
H_{*}\left(\Omega \Sigma C P^{\infty} ; R\right) \cong H_{*}(M \xi ; R),
$$

the generator $Z_{i}$ corresponds to an element $z_{i} \in H_{2 i}\left(M \xi ; R\right.$ ) (we set $z_{0}=1$ ). Thomifying the map $i: C P^{\infty} \longrightarrow \Omega \Sigma C P^{\infty}$, we obtain a map $M i: \Sigma^{\infty} M U(1)$ $\longrightarrow \Sigma^{2} M \xi$ and it is easy to see that

$$
M i_{*} \beta_{i+1}=z_{i} .
$$

Working in mod $p$ homology and using the standard formula for the (left) coaction $\psi[2]$, we obtain

$$
\sum_{i \geqslant 0} \psi\left(z_{i}\right) t^{i+1}=\sum_{j \geqslant 0} \xi(t)^{j+1} \otimes z_{j}
$$

where

$$
\xi(t)=\sum_{k \geqslant 0} \xi_{k} t^{p^{k}} .
$$


We now introduce elements $w_{i} \in H_{2 i}(M \xi ; R)$ by requiring that they satisfy the functional equation

$$
\sum_{i \geqslant 0} z_{i}\left(\sum_{j \geqslant 0} w_{j} t^{j+1}\right)^{i+1}=t,
$$

where we treat $t$ as a variable that commutes with everything in sight. This amounts to an infinite sequence of equations of the general form

$$
w_{n}+\cdots+z_{n}=0 \quad(n \geqslant 1),
$$

where the intermediate terms involve the elements $z_{i}, w_{i}$ with $i=1, \ldots, n-1$ and $w_{0}=1$. Hence we can recursively solve this system of equations for the $w_{n}$ and the solution is obviously unique. Mapping into $H_{*}(M U ; R)$ we obtain the usual generators $m_{i} \in H_{2 i}(M U ; R)$ described in [2] and these can be expressed explicitly using the Lagrange inversion formula. But it is not obvious in what sense such a formula exists within the non-commutative context where we are working.

Now we focus on the case of $H_{*}\left(M \xi ; \mathrm{F}_{p}\right)$ and investigate the $\mathscr{A}_{*}$-coaction $\psi$ on the elements $w_{i}$. We will make heavy use of the fact that $\psi$ is multiplicative, i.e., for homogeneous elements $u \in H_{2 r}\left(M \xi ; \mathrm{F}_{p}\right)$ and $v \in H_{2 s}\left(M \xi ; \mathrm{F}_{p}\right)$ with

$$
\begin{aligned}
& \psi(u)=\sum_{i} x_{i} \otimes u_{i} \in \mathscr{A}_{*} \otimes H_{*}\left(M \xi ; \mathrm{F}_{p}\right), \\
& \psi(v)=\sum_{j} y_{j} \otimes v_{j} \in \mathscr{A}_{*} \otimes H_{*}\left(M \xi ; \mathrm{F}_{p}\right),
\end{aligned}
$$

where $u_{i}, v_{j} \in H_{*}\left(M \xi ; \mathrm{F}_{p}\right)$ and $x_{i}, y_{j} \in \mathscr{A}_{*}$, we have

$$
\psi(u v)=\sum_{i, j} x_{i} y_{j} \otimes u_{i} v_{j} \in \mathscr{A}_{*} \otimes H_{*}\left(M \xi ; \mathrm{F}_{p}\right) .
$$

Let us write $\widetilde{w}_{i}=\psi\left(w_{i}\right)$ and

$$
\widetilde{w}(t)=\sum_{i \geqslant 0} \widetilde{w}_{i} t^{i+1} \in\left(\mathscr{A}_{*} \otimes H_{*}\left(M \xi ; \mathrm{F}_{p}\right)\right)[[t]] .
$$

Then from (7.2) we have

$$
\sum_{i \geqslant 0}\left(1 \otimes z_{i}\right)[(\xi \otimes 1)(\widetilde{w}(t))]^{i+1}=t,
$$


where $t$ and all elements of $\mathscr{A}_{*} \otimes 1$ commute with everything. Of course,

$$
(\xi \otimes 1)(\widetilde{w}(t))=\sum_{r \geqslant 0}\left(\xi_{r} \otimes 1\right)(\widetilde{w}(t))^{p^{r}}
$$

Now unravelling the coefficients we find that the $\widetilde{w}_{i}$ satisfy a sequence of equations of same general form as (7.3):

$$
\widetilde{w}_{n}+\cdots+1 \otimes z_{n}=0 \quad(n \geqslant 1),
$$

and clearly this has a unique solution. Now consider the series

$$
(\zeta \otimes 1)(1 \otimes w)(t)=\sum_{r \geqslant 0}\left(\zeta_{r} \otimes 1\right)[(1 \otimes w)(t)]^{p^{r}} .
$$

Using (7.2), we obtain

$$
\begin{aligned}
\sum_{i \geqslant 0}\left(1 \otimes z_{i}\right)[(\xi \otimes 1)(\zeta \otimes 1)(1 \otimes w(t))]^{i+1} & =\sum_{i \geqslant 0}\left(1 \otimes z_{i}\right)\left(1 \otimes w(t)^{i+1}\right) \\
& =\sum_{i \geqslant 0} 1 \otimes z_{i} w(t)^{i+1}=t
\end{aligned}
$$

hence we have

$$
\sum_{i \geqslant 0} \psi\left(w_{i}\right) t^{i+1}=\sum_{j \geqslant 0} \zeta_{j} \otimes w(t)^{p^{j}}
$$

This has the same form as the coaction on the $m_{i}$ in $H_{*}\left(M U ; \mathrm{F}_{p}\right)$ as given in [2], although the non-commutativity means that the explicit formulae for $\psi\left(w_{n}\right)$ are more complicated. In particular, the simple formula

$$
\psi\left(m_{p^{r}-1}\right)=\sum_{0 \leqslant i \leqslant r} \zeta_{i} \otimes m_{p^{r-i}-1}^{p^{i}}
$$

is replaced by one involving many more terms.

\section{The homology of $\mathrm{THH}(\mathrm{M \xi})$}

The following is a description of the result of applying the Bökstedt spectral sequence to $M \xi$ and of some things we learned from [6]. In his influential unpublished preprint [7] (see for instance [22,3.1] for a published account), Marcel Bökstedt developed a spectral sequence for calculating the homology of the $T H H$-spectrum of a strictly associative spectrum. 
The spectral sequence for the integral homology of the associative spectrum $M \xi$ has as its $\mathrm{E}^{2}$-page the Hochschild homology of $H_{*}(M \xi)$, so we have

$$
\mathrm{E}_{s, t}^{2}=H H_{s, t}\left(H_{*}(M \xi)\right) \Longrightarrow H_{s+t}(T H H(M \xi)) .
$$

Now $H_{*}(M \xi)$ is a tensor algebra $T V$, where $V$ is the free Z-module generated by the elements $z_{i}$ for $i \geqslant 1$, thus $H_{*}(M \xi)$ is free as an associative algebra and the Hochschild homology vanishes except for homological degrees zero and one. Moreover,

$$
\begin{aligned}
& H H_{0}\left(H_{*}(M \xi)\right)=\bigoplus_{m \geqslant 0} V^{\otimes m} / C_{m}, \\
& H H_{1}\left(H_{*}(M \xi)\right)=\bigoplus_{m \geqslant 1}\left(V^{\otimes m}\right)^{C_{m}},
\end{aligned}
$$

where $V^{\otimes m} / C_{m}$ and $\left(V^{\otimes m}\right)^{C_{m}}$ denote the coinvariants and invariants respectively for the action of the cyclic group $C_{m}$. As there are just two non-trivial columns, this spectral sequence collapses placing the part arising as the coinvariants in total even degree and the part coming from the invariants in total odd degree. We note that for each degree $m$, a basis of $V^{\otimes m}$ consists of the tensors of length $m$ in the $z_{i}$ and this is a permutation basis for the action of $C_{m}$. Therefore $V^{\otimes m}$ decomposes as a direct sum of $C_{m}$-modules corresponding to the orbits of the action and for each of these it is straightforward to see that the coinvariants and invariants are both free of rank one over Z. Hence $H H_{0}\left(H_{*}(M \xi)\right)$ and $H H_{1}\left(H_{*}(M \xi)\right)$ are finitely generated free abelian groups in each bidegree and therefore $H_{*}(T H H(M \xi))$ consists of such groups in each degree.

Andrew Blumberg, Ralph Cohen and Christian Schlichtkrull [6] give a nice description of topological Hochschild homology of Thom spectra. Let $B F$ denote the classifying space for spherical fibrations. Given a based map $f: X \longrightarrow \mathrm{B} B F$, which induces a loop map from the based loop space $\Omega X$ to $B F$, they describe $T H H(M f)$ as the Thom spectrum associated to the composite

$$
L X \longrightarrow L \mathrm{~B} B F \simeq B F \times \mathrm{B} B F \stackrel{\mathrm{id} \times \eta}{\longrightarrow} B F \times B F \longrightarrow B F .
$$

Here, $L(-)$ denotes the free loop space on a space. In particular, the homology of $T H H(M f)$ is the homology of the free loop space on $X$ if we actually start with a map to $B S F$.

Let ad: $\Sigma \mathrm{C} P^{\infty} \longrightarrow \mathrm{B} B U$ be the adjoint of the inclusion $j: C P^{\infty}=$ $B U(1) \longrightarrow B U$. 
In our case, the map to $B F$ factors over $B U$ and thus over $B S F$ and we obtain that $T H H(M \xi)$ is the Thom spectrum associated to

$$
L \Sigma C P^{\infty} \stackrel{L a d}{\longrightarrow} L B B U=L(S U) \simeq \Omega S U \times S U=B U \times S U \longrightarrow B U
$$

because $\eta$ becomes null homotopic here.

Goodwillie's result [13, Theorem V.1.1] identifies the $S^{1}$-equivariant homology of the free loop space with cyclic homology of the chains on the based (Moore) loop space. The corresponding result for Hochschild homology reads in our case

$$
H_{*}\left(L \Sigma C P^{\infty}\right) \cong H H_{*}\left(C_{*}\left(\Omega \Sigma C P^{\infty}\right)\right) .
$$

Thus the $\mathrm{E}^{2}$-term of associated hyperhomology spectral sequence has the same form as that of the Bökstedt spectral sequence. We give a general comparison result for this situation.

Let $X=\Omega Y$ be a loop space with torsion-free homology and with a loop map $f: X \longrightarrow B S F$. Assume that $Y$ is path connected and well-pointed. We can consider the associated Bökstedt and hyperhomology spectral sequences for $X$ and its Thom spectrum $M f$.

Proposition 8.1. There is an isomorphism between the hyperhomology spectral sequence and the Bökstedt spectral sequence induced by an isomorphism of exact couples.

Proof. For the hyperhomology spectral sequence we have to consider the bicomplex with $(p, q)$-term $\left(\bar{C}_{q}\left(C_{*}(\Omega Y)\right)\right)_{p}$ where $\bar{C}_{*}$ denotes the reduced Hochschild complex. We take the $s$-filtration of the total complex by considering

$$
F_{S}(\text { hyper })_{n}=\bigoplus_{\substack{p+q=n \\ q \leqslant s}}\left(\bar{C}_{q}\left(C_{*}(\Omega Y)\right)\right)_{p} .
$$

The corresponding exact couple then consists of the terms

$$
\begin{aligned}
D(\text { hyper })_{s, t} & =H_{s+t} F_{s}(\text { hyper })_{*}, \\
E(\text { hyper })_{s, t} & =H_{s+t}\left(F_{s}(\text { hyper })_{*} / F_{s-1}(\text { hyper })_{*}\right)=\left(\bar{C}_{s}\left(H_{*}(\Omega Y)\right)\right)_{t} .
\end{aligned}
$$

For the Bökstedt spectral sequence, we use the skeletal filtration of $T H H(M f)$. This gives rise to another exact couple with

$$
D(\mathrm{Bö})_{s, t}=H_{s+t}\left(T H H^{[s]}(M f)\right),
$$

in which $T H H^{[s]}(M f)$ denotes the $s$-skeleton of the realization of the simplicial spectrum $T H H(M f)$. Since topological Hochschild homology gives rise to a 
proper simplicial spectrum we can apply [11, X.2.9] in order to identify the filtration quotients and get

$$
E(\mathrm{Bö})_{s, t} \cong H_{s+t}\left(\Sigma^{s}(M f \wedge \underbrace{\overline{M f} \wedge \cdots \wedge \overline{M f}}_{s}) .\right.
$$

Here, $\overline{M f}$ is the cofibre of $\mathrm{S} \longrightarrow M f$. Therefore we obtain

$$
E(\mathrm{Bö})_{s, t} \cong\left(\bar{C}_{s}\left(H_{*}(M f)\right)\right)_{t} .
$$

The Thom isomorphism yields an isomorphism between the two exact couples and its multiplicativity ensures that the higher differentials are preserved as well.

\section{REFERENCES}

1. Adams, J. F., Lectures on generalised cohomology, 1969 Category Theory, Homology Theory and their Applications, III (Battelle Institute Conference, Seattle, Wash., 1968, Vol. Three), Lecture Notes in Math. 99 (1969), 1-138.

2. Adams, J. F., Stable homotopy and generalised homology, Chicago Lectures in Math. (1974).

3. Arthan, R. D., Bullett, S. R., The homology of $M \mathrm{O}(1)^{\wedge \infty}$ and $M \mathrm{U}(1)^{\wedge \infty}$, J. Pure Appl. Algebra 26 (1982), 229-234.

4. Baker, A., Husemoller-Witt decompositions and actions of the Steenrod algebra, Proc. Edinburgh Math. Soc. (2) 28 (1985), 271-288.

5. Baker, A. J., May, J. P., Minimal atomic complexes, Topology 43 (2004), 645-665.

6. Blumberg, A., Cohen, R. L., Schlichtkrull, C., Topological Hochschild homology of Thom spectra, in preparation.

7. Bökstedt, M., Topological Hochschild homology, preprint.

8. Crossley, M. D., The Steenrod algebra and other copolynomial Hopf algebras, Bull. London Math. Soc. 32 No. 5 (2000), 609-614.

9. Ditters, E. J., Curves and formal (co) groups, Invent. Math. 17 (1972), 1-20.

10. Ditters, E. J., Scholtens, A. C. J., Free polynomial generators for the Hopf algebra QSymm of quasisymmetric functions, J. Pure Appl. Algebra 144 No. 3 (1999), 213-227.

11. Elmendorf, A. D., Kriz, I., Mandell, M. A., May, J. P., Rings, modules, and algebras in stable homotopy theory, Math. Surveys Monogr. 47 (1997).

12. Fresse, B., On the homotopy of simplicial algebras over an operad, Trans. Amer. Math. Soc. 352 (2000), 4113-4141.

13. Goodwillie, T. G., Cyclic homology, derivations, and the free loopspace, Topology 24 No. 2 (1985), 187-215.

14. Gray, B., Ray, N., Splitting $C \mathrm{P}^{\infty}$ and $B Z / p^{n}$ into Thom spectra, Math. Proc. Cambridge Philos. Soc. 106 (1989), 263-271.

15. Hazewinkel, M., Formal groups and applications, Pure Appl. Math. 78 (1978).

16. Hazewinkel, M., The algebra of quasi-symmetric functions is free over the integers, Adv. Math. 164 (2001), 287-300.

17. Hazewinkel, M., Symmetric functions, noncommutative symmetric functions, and quasisymmetric functions, Acta Appl. Math. 75, No. 1-3, (2003), 55-83.

18. Hazewinkel, M., Symmetric functions, noncommutative symmetric functions and quasisymmetric functions. II, Acta Appl. Math. 85, No. 1-3, (2005), 319-340. 
19. Hazewinkel, M., The primitives of the Hopf algebra of noncommutative symmetric functions, preprint, math.QA/0410365.

20. Husemöller, D., The structure of the Hopfalgebra $H_{*}(B U)$ over a $\mathrm{Z}_{(p)}$-algebra, Amer. J. Math. 93 (1971), 329-349.

21. Liulevicius, A., Arrows, symmetries and representation rings, J. Pure Appl. Algebra 19 (1980), 259-273.

22. McClure, J. E., Staffeldt, R. E., On the topological Hochschild homology of bu, I, Amer. J. Math. 115 (1993), 1-45.

23. McGibbon, C. A., Stable properties of rank 1 loop structures, Topology 20 (1981), 109-118.

24. Milnor, J. W., Moore, J. C., On the structure of Hopf algebras, Ann. of Math. (2) 81 (1965), 211-264.

25. Morava, J., Toward a Fundamental Groupoid for the Stable Homotopy Category, Geom. Topol. Monogr. 10 (2007), 293-317.

26. Morisugi, K., Projective elements in $K$-theory and self maps of $\Sigma C P^{\infty}$, J. Math. Kyoto Univ. 38 (1998), 151-165.

27. Pilz, G., Near-rings. The Theory and its Applications, revised edition, North-Holland Publishing Company (1983).

28. Reutenauer, C., Free Lie Algebras, London Math. Soc. Monogr. (N.S.) 7 (1993).

29. Scheerer, H., On rationalized $H$ - and co-H-spaces, with an appendix on decomposable Hand co-H-spaces, Manuscripta Math. 51 (1985), 63-87.

30. Selick, P., Introduction to Homotopy Theory, Fields Inst. Monogr. 9 (1997).

31. Selick, P., Theriault, S. D., Wu, J., Decompositions of H-spaces, in preparation.

32. Switzer, R. M., Algebraic Topology—Homotopy and Homology, reprint of the 1975 original, Classics Math. (2002).

33. Theriault, S. D., Homotopy decomposition involving the loops of coassociative Co-H-spaces, Canad. J. Math. 55 (2003), 181-203.

34. Whitehead, G. W., Elements of Homotopy Theory, Grad. Texts Math. (1978).

DEPARTMENT OF MATHEMATICS

UNIVERSITY OF GLASGOW

GLASGOW G12 8QW

SCOTLAND

E-mail: a.baker@maths.gla.ac.uk

URL: http://www.maths.gla.ac.uk/ $/ a j b$
DEPARTMENT MATHEMATIK

DER UNIVERSITÄT HAMBURG

20146 HAMBURG

GERMANY

E-mail: richter@math.uni-hamburg.de

URL: http://www.math.uni-hamburg.de/home/richter/ 